Innovative Applications of O.R.

\title{
Centralized and decentralized management of groundwater with multiple users
}

\author{
Yahya Saleh ${ }^{\mathrm{a}}$, Ülkü Gürler ${ }^{\mathrm{a}, *}$, Emre Berk ${ }^{\mathrm{b}}$ \\ ${ }^{a}$ Department of Industrial Engineering, Bilkent University, 06800 Ankara, Turkey \\ ${ }^{\mathrm{b}}$ Department of Management, Bilkent University, 06800 Ankara, Turkey
}

\section{A R T I C L E I N F O}

\section{Article history:}

Received 9 November 2009

Accepted 26 May 2011

Available online 2 June 2011

\section{Keywords:}

OR in natural resources

Game theory

Water resources management

Darcy's Law

\begin{abstract}
A B S T R A C T
In this work, we investigate two groundwater inventory management schemes with multiple users in a dynamic game-theoretic structure: $(i)$ under the centralized management scheme, users are allowed to pump water from a common aquifer with the supervision of a social planner, and (ii) under the decentralized management scheme, each user is allowed to pump water from a common aquifer making usage decisions individually in a non-cooperative fashion. This work is motivated by the work of Saak and Peterson [14], which considers a model with two identical users sharing a common aquifer over a two-period planning horizon. In our work, the model and results of Saak and Peterson [14] are generalized in several directions. We first build on and extend their work to the case of $n$ non-identical users distributed over a common aquifer region. Furthermore, we consider two different geometric configurations overlying the aquifer, namely, the strip and the ring configurations. In each configuration, general analytical results of the optimal groundwater usage are obtained and numerical examples are discussed for both centralized and decentralized problems.
\end{abstract}

(c) 2011 Elsevier B.V. All rights reserved.

\section{Introduction}

Effective management of limited resources shared by multiple users is becoming of more importance due to increasing pressures resulting from demographic and/or economic growth and ecological deterioration. Such resources include fisheries, water and clean air. They suffer from either lack of enforceable private property rights or their designation of common/public property. Furthermore, these resources exhibit an interesting property. They tend to move from one location to another depending on the extent of usage. Underground water laterally flows within an aquifer along with the hydrological gradient (difference between low and high water levels); schools of fish travel to other locations to run away from heavy fishing in one location; pollution at a point is dissipated degrading the overall quality over a larger area. This property permits gaming behavior among users. In this paper, we focus on groundwater as one of a number of limited resources. Our motivation comes from the following. Scarcity of water - for personal and industrial/agricultural use - is increasing in both absolute and relative terms. Shortages observed in rainfall, adverse micro-climatic changes, contamination of groundwater reservoirs (aquifers) due to increasing industrial and human pollution result in a decrease in the amount of water of certain quality fit for use. Increases in demand for water due to growth in the overall popu-

\footnotetext{
* Corresponding author. Tel.: +90 3122901520.

E-mail addresses: saleh@bilkent.edu.tr (Y. Saleh), ulku@bilkent.edu.tr (Ü. Gürler), eberk@bilkent.edu.tr (E. Berk).
}

lations and changes in consumption patterns result in the relative scarcity of this precious resource. In arid and semi-arid regions of the globe, the scarcity is reaching critical levels. The gaming behavior of users may be detrimental for many communities for some generations to come.

Earlier works on groundwater management have argued that welfare gain from applying different policies and disadvantages of gaming behavior are likely to be negligible. Specifically, Gisser and Sanchez [7] have shown that, if the common and freely accessed aquifer's storage capacity is large enough, a free market (decentralized) behavior and optimal centralized control strategies perform equally well in terms of the welfare gain from groundwater usage. Allen and Gisser [1] extended their work by considering a non-linear demand function. They also confirmed that if water rights are properly defined and if the aquifer's storage capacity is large, then the difference between no control strategy and optimal control strategy is small, and, thus, can be ignored for practical policy considerations. However, Negri [11] objected to these findings on the grounds that the fundamental assumption of openly accessed groundwater aquifer is not valid. An open access aquifer assumes that the overlying users can use as much of it as they wish regardless of their location. However, for most aquifers, this is not possible since access to groundwater is usually limited since not all users own the overlying land as well as the water rights. Furthermore, the lateral flows within the aquifer are not instantaneous. Saak and Peterson [14] address these inadequacies by considering a game-theoretic restricted access aquifer with identical users over a finite planning horizon (of two periods), 
where lateral non-instantaneous flows are governed by a natural law. Their contribution is twofold: they model underground hydrological behavior more realistically and they incorporate possibility of lack of information about the ground transmissivity by users. However, their analysis is restricted to two identical users.

In this work, we build on their model and extend it to the case of multiple non-identical users with two different geometric configurations overlying the aquifer. We investigate two groundwater inventory management schemes with multiple users in a dynamic game-theoretic structure: $(i)$ under the centralized management scheme, users are allowed to pump water from a common aquifer with the supervision of a social planner, and (ii) under the decentralized management scheme, each user is allowed to use water from a common aquifer making usage decisions individually in a non-cooperative fashion. In our work, the model and results of Saak and Peterson [14] are generalized in several directions. We first extend their work to the multiple non-identical user setting with two different geometric configurations overlying the aquifer, namely, the strip and ring configurations. The non-identical structure among users is represented in the differences of the parameters of each user's profit function. The rationale behind our extension to both strip and ring configurations naturally arise when there are more than two users, since with two users they are identical. In fact, this resulting variation in the configuration types itself motivates the extension to more than two users. Apart from geometric description, it is more important to note that the strip and ring structures mainly differ in the number of neighboring users that each user interacts, where in the latter one each user interacts with two neighbors. In each configuration, namely, in both the decentralized and centralized problems, general analytical results of the optimal water usage are obtained. We are able to obtain closed form optimal solutions for special cases of parameter values. Our results reduce to those of Saak and Peterson [14] in the case of two identical users, and validate some of their conjectures about multiple users.

In our study, we show the existence of a unique Nash equilibrium in both configurations and provide the solution structure for the decentralized problems with $n$ non-identical users. For identical users, we also manage to derive explicit solutions for the optimal water usage. It is shown that in strip configuration with $n$ identical users, the optimal Nash equilibrium usage quantities oscillate about the optimal Nash equilibrium usage quantities of the ring configuration. Our numerical results indicate that as the underground water transmission coefficient increases, users become more greedy and tend to use more water. The analysis for the centralized problem in the strip and ring configurations reveals that the optimal solution of groundwater usage is symmetric, unique across users and independent of the characteristics of the groundwater aquifer. This generalizes one of the important findings of Saak and Peterson [14] regarding the optimal equilibrium water usage. An important question that might be raised by a policy maker is about the possibility of coordinating the groundwater system by achieving the centralized solution in the decentralized game theoretic setting via a single pricing mechanism. Our results show that in both configurations, this is impossible to be realized. Additionally, we consider a variant of our model with salvage possibility for left over water as a proxy for extending the problem horizon.

Our study focuses on the optimal water consumption of multiple users with lateral transmissivity of groundwater among adjacent users under centralized and decentralized management schemes. Another work that considers centralized water management under a different setting is by Haouari and Azaiez [8]. Their work differs from ours in that the decision-maker (local authorities) aims at selecting crops and allocating water and land to them in order to maximize the total linear profit obtained from annual and seasonal crops for the whole year of the plan. Their model is addressed centrally for a limited availability of water stock without allowing any "commonality" of the water source which creates a gaming behavior in the model. In our work, because of the aquifer's commonality, a strategic-form game arises between the $n$ - nonidentical users, and, hence, the water management problem is addressed centrally and decentrally. Also, our profit function is of quadratic form. There are also a number of other studies in literature that consider conjunctive use of multiple water resources such as Azaiez and Hariga [2], Azaiez [3] and Azaiez et al. [4]. Other related studies on water management and operations models can be found in Yeh [15] and Labadie [10].

The rest of the paper is organized as follows. Section 2 includes the preliminaries and the specifics of the model. Section 3 presents the analytical results of the two water management schemes for the strip configuration, while those related to the ring configuration are presented in Section 4. Numerical results are presented in Section 5. Section 6 concludes our work.

\section{Preliminaries and basic model properties}

In this section, we lay out some common assumptions and model properties in our analysis. We consider a system of $n$ users who are non-identical in their characteristics configured over and using a common groundwater aquifer to maximize their profits discounted over a finite planning horizon of two periods in either a centralized or decentralized manner. User $i$ has access to an underground water stock of $x_{i, t}$ at the beginning of period $t$, for $i=1, \ldots, n$ and $t=1,2$. There is also an aquifer recharge $w_{i, 1}=w_{1}$ for all $i$ at the beginning of Period 2; we assume that recharge does not alleviate the underground water level above the base level $x_{i, 0}$. We allow the cost and revenue parameters to vary over time among users. Let $u_{i, t}$ denote the amount of groundwater pumped (and used) by user $i$, $i=1, \ldots, n$, in period $t, t=1,2$. It is assumed that $u_{i, t} \leqslant x_{i, t}$, which implies that groundwater is essentially a private resource within each period and a user can not access groundwater lying beneath another user. As water levels change locally due to consumption by each user, water in the aquifer may flow laterally between adjacent users (between the adjacent areas corresponding to the users' plots). The inter-period lateral flow of groundwater between adjacent users is governed by Darcy's Law. This natural law states that the rate of flow of groundwater through a certain medium (soil) is proportionally related to the hydrologic gradient (i.e. the driving force acting on water) and the conductivity of the medium (i.e. the measure of the ability of medium to transmit water), $\alpha$, as stated in Hornberger et al. [9]. The water stock level of a user in a period will be expressed as a function of the previous period's stock level of the user, the groundwater usage of the user and the neighbors, as well as the aquifer's hydrological properties. In the analysis below, we assume that initial water stocks $x_{i, 1}$, are identical for all users $i=1, \ldots, n$; furthermore, the soil properties are assumed similar so that all users' water stocks are subject to the same $\alpha$. The interaction in the availabilities of groundwater stocks among users makes their decentralized and centralized problems nonseparable.

The profit functions of users are of quadratic form similar to Saak and Peterson [14]. The profit function of groundwater usage realized by user $i$ for time period $t$ is given by

$g_{i, t}\left(u_{i, t}, x_{i, t}\right)=\left[\rho_{i, t} a_{i, t}-c_{i, t}\left(x_{i, 0}-x_{i, t}\right)\right] u_{i, t}-0.5\left(\rho_{i, t} b_{i, t}+c_{i, t}\right) u_{i, t}^{2}$,

where $x_{i, 0}=x_{i, 1}$ and the cost-revenue parameters $\rho_{i, t}, a_{i, t}, b_{i, t}, c_{i, t}>0$ and satisfy the following condition

$\left(\rho_{i, t} b_{i, t}+c_{i, t}\right) x_{i, 0}<\rho_{i, t} a_{i, t}<\left(2 \rho_{i, t} b_{i, t}+c_{i, t}\right) x_{i, 0}$.

The condition in Eq. (2) on the parameters follows from the models in Saak and Peterson [14] and is needed for some of our structural 
results herein as for theirs. In the context of agricultural water usage, it is assumed that the pumped underground water is used for irrigation of crops. The profit function in (1) is a special case of the general profit function $v_{i, t}\left(\rho_{i, t} y_{i, t}\left(u_{i, t}\right)-\tau_{i, t}\left(u_{i, t}, x_{i, t}\right)-k_{i, t}\right)$, which has an empirical estimated specification in Peterson and Ding [13], where $v_{i, t}$ is utility-of-income function, $\rho_{i, t}$ is the price per unit of the crop, $y_{i, t}$ is the yield of the crop which is dependent on the amount of water used, $\tau_{i, t}\left(u_{i, t}, x_{i, t}\right)$ is the cost of pumped groundwater (a joint function of water usage and groundwater stock level) and $k_{i, t}$ is the fixed cost of infrastructural (farming) inputs. When we assume a linear utility-of-income function, $\left(v_{i, t}(z)=z\right)$, a quadratic yield function $y_{i, t}\left(u_{i, t}\right)=a_{i, t} u_{i, t}-0.5 b_{i, t} u_{i, t}^{2}$, a quadratic groundwater extraction cost $\tau_{i, t}\left(u_{i, t}, x_{i, t}\right)=c_{i, t}\left[\left(x_{i, 0}-x_{i, t}\right) u_{i, t}+0.5 u_{i, t}^{2}\right]$ and omit the fixed costs $\left(k_{i, t}=0\right)$, we get the profit function in Eq. (1). For this profit expression, we have the following key property.

Lemma 1. Positivity, continuity, concavity

(i) For $u_{i, t} \leqslant x_{i, t} \leqslant x_{i, 0}$, the function $g_{i, t}\left(u_{i, t}, x_{i, t}\right)$ is strictly increasing in $u_{i, t}, i=1, \ldots, n, t=1,2$.

(ii) The function $g_{i, t}\left(u_{i, t}, x_{i, t}\right)$ is continuous and concave in $u_{i, t}$, $i=1, \ldots, n, t=1,2$.

Proof. All proofs are provided in the Online Supplement.

We construct our models with non-identical users in the general case. The differences among users may be due to differences in the yield and cost parameters of the users. The differences in the yield parameters $\left(\rho_{i, t}, a_{i, t}\right.$ and $b_{i, t}$ ) among users represent different cropping and irrigation patterns adopted by users, whereas the difference in the cost parameters $\left(c_{i, t}\right.$ and $\left.k_{i, t}\right)$ represents different technologies and machinery utilized in pumping groundwater from the common aquifer and in irrigating the grown crops. The geography of the aquifer region and the soil properties (hydrology) of the land being planted and irrigated characterize possible different transmission structures for the users configured over the common aquifer. Additionally, the specific configuration of the users over this aquifer contribute to the water dynamics over time among users. In this work, we consider two configurations - the strip and ring configurations - within the general framework as outlined above.

\section{Strip Configuration}

We consider the system of $n$ non-identical users distributed adjacently in a strip over the common groundwater aquifer. The setting may be envisioned as an abstraction of a more complex geographic configuration with the only restriction that each user has at most two neighbors. For one dimensional flow of groundwater, there will be lateral flow of groundwater among adjacently located users. Then, the extreme users on the strip (the first and the last) will receive groundwater flow only from one neighbor, whereas for all other (non-extreme) users, flow will be from the two neighbors on both sides. Hence, for $i=1$ and $j=2$ and, $i=n$ and $j=n-1$, the lateral flow of groundwater in period 1 is given by $Q_{j, i}=-\alpha\left[\left(x_{i, 1}-u_{i, 1}+w_{i, 1}\right)-\left(x_{j, 1}-u_{j, 1}+w_{j, 1}\right)\right]=\alpha\left(u_{i, 1}-u_{j, 1}\right)$, where $\alpha \in[0,0.5]$ is the lateral flow (aquifer transmissivity) coefficient, summarizing the hydrologic dynamics of the groundwater aquifer, and $\left(x_{i, 1}-u_{i, 1}+w_{i, 1}\right)-\left(x_{j, 1}-u_{j, 1}+w_{j, 1}\right)$ is the hydrologic gradient (the difference in hydrologic head between the wells). Similarly, by applying Darcy's Law in period 1 , a non-extreme user $i, i=1, \ldots, n-1$, would have lateral inflows $Q_{i-1, i}$ and $Q_{i+1, i}$, where $Q_{i-1, i}=\alpha\left(u_{i, 1}-u_{i-1,1}\right)$ and $Q_{i+1, i}=\alpha\left(u_{i, 1}-u_{i+1,1}\right)$. In this configuration, we consider below the two kinds of decision making - decentralized and centralized problems.

\subsection{The decentralized problem}

In the decentralized problem, each user has the objective of maximizing his/her own total discounted profit over the horizon of two periods by choosing the water usage quantity in each period. But, at the same time, each user has to take into account usages of all other users due to the commonality of the underground aquifer. This generates an $n$-player normal-form game, where the water usage quantity in each period is the strategy of a player (a user), and the payoff function is given by a user's expected total discounted profit over the horizon. The strategy space of any user is constructed from the other users' decisions of water usage and the available (and finite) underground water stocks in any period. In this section, we consider this game-theoretic model and investigate its properties. The decentralized problem above can be stated formally as a dynamic program as follows. Let $\Gamma_{i, t}^{*}\left(\vec{u}_{t}, \vec{x}_{t}\right)$ denote the maximum expected total profit under an optimal water usage schedule for user $i$ for periods $t$ through the end of horizon, where $\vec{u}_{t}=\left(u_{1, t}, \ldots, u_{n, t}\right)^{T}$ is the water usage vector for all users in period $t$ and $\vec{x}_{t}=\left(x_{1, t}, \ldots, x_{n, t}\right)^{T}$ is the water stock vector for all users at the beginning of period $t$. For $t=1,2$, the decentralized problem of user $i, i=1, \ldots, n$, is solved by the following dynamic program

$$
\begin{aligned}
& \Gamma_{i, t}^{*}\left(\vec{u}_{t}, \vec{x}_{t}\right)= \max _{u_{i, t}} \Gamma_{i, t}\left(\vec{u}_{t}, \vec{x}_{t}\right) \\
&= \max _{u_{i, t}}\left[g_{i, t}\left(u_{i, t}, x_{i, t}\right)+\beta_{i, t} \Gamma_{i, t+1}^{*}\left(\vec{u}_{t+1}, \vec{x}_{t+1}\right)\right], \\
& \text { s.t. } x_{i, t+1}=\left\{\begin{array}{c}
x_{i, t}+w_{i, t}-(1-\alpha) u_{i, t}-\alpha u_{j, t}, \\
(i, j) \in\{(1,2),(n, n-1)\} \\
x_{i, t}+w_{i, t}-(1-2 \alpha) u_{i, t}-\alpha\left(u_{i-1, t}+u_{i+1, t}\right), \\
i=2, \ldots, n-1,
\end{array}\right.
\end{aligned}
$$

$0 \leqslant u_{i, t} \leqslant x_{i, t}$

In the above problem, the decision variables for this simultaneous optimization problem are the water usage quantities of each user in each period, $u_{i, t}$. Eq. (4) corresponds to the recursive temporal relationship among the water stocks of the users as dictated by Darcy's Law. In our formulation, we assume the same hydrological transmissivity coefficient $\alpha$ across the strip for all users and all periods, as it would be the case for short time horizons. Eq. (5) gives the constraint for each user's water usage. We assume that the discount rate $\beta_{i, t}=\beta$ with $0 \leqslant \beta \leqslant 1$; and set $x_{i, 1}=x_{1}$, $w_{i, 1}=w_{1}$ and $\Gamma_{i, 3}^{*}\left(\vec{u}_{3}, \vec{x}_{3}\right) \equiv 0$ for all $\vec{x}_{3}, \vec{u}_{3}$ and for $i=1, \ldots, n$. (We later relax the condition on $\left.\Gamma_{3}^{*}\left(\vec{u}_{3}, \vec{x}_{3}\right)\right)$. We are now ready to examine some properties of the optimal solution to the above formulation. We first provide the structural results for the objective function, $\Gamma_{i, t}\left(\vec{u}_{t}, \vec{x}_{t}\right)$. From Lemma 1 (i), immediately we have the following.

Corollary 1. The within-period profit function $g_{i, t}\left(u_{i, t}, x_{i, t}\right)$ attains its maximum at $u_{i, t}^{*}=x_{i, t}, i=1, \ldots, n, t=1,2$.

This result has two implications. (i) The myopic solution of the problem is trivial; that is, all water resources are depleted in the first period for any length of the horizon. (ii) In the optimal solution, all users deplete their water resources in the very last period, (i.e., $\left.u_{i, 2}^{*}=x_{i, 2}, \forall i\right)$. Therefore, we have $\Gamma_{i, 1}\left(\vec{u}_{1}, \vec{x}_{1}\right)=$ $\left[g_{i, 1}\left(u_{i, 1}, x_{i, 1}\right)+\beta g_{i, 2}\left(x_{i, 2}, x_{i, 2}\right)\right]$. Furthermore, $x_{i, 2}$ is a function of $\vec{u}_{1}$; and, hence, the $n$ - user problem given in (3)-(5) reduces to a single period problem which is only a function of $\vec{u}_{1}$ and $\vec{x}_{1}$. We can use these implications to obtain below a tighter formulation of the original problem and to establish additional properties. 
Proposition 1. Positivity, continuity, concavity

(i) $\Gamma_{i, 1}\left(\vec{u}_{1}, \vec{x}_{1}\right)$ is strictly increasing in $u_{i, 1}$ at $u_{i, 1}=0$ if $\rho_{i, 1} a_{i, 1} \geqslant$ $\beta\left(\rho_{i, 2} a_{i, 2}+c_{i, 2} w_{1}\right), i=1, \ldots, n$.

(ii) $\Gamma_{i, 1}\left(\vec{u}_{1}, \vec{x}_{1}\right)$ is continuous and jointly concave in $\vec{u}_{1}$ if and only if $c_{i, 2} \leqslant \rho_{i, 2} b_{i, 2}, i=1, \ldots, n$.

The first part of the above result establishes the positivity of the optimal solution, that is $u_{i, 1}^{*}>0$ for all $i, i=1, \ldots, n$. Therefore, it suffices for our setting to consider a tighter search space $\left(0<u_{i, 1} \leqslant x_{1}\right.$ $\forall i$ ). The latter part guarantees a well-behaving objective function for optimization. We can now re-state the two-period decentralized problem as follows. For $i=1, \ldots, n$,

$\max _{u_{i, 1}} \Gamma_{i, 1}\left(\vec{u}_{1}, \vec{x}_{1}\right)=\max _{u_{i, 1}}\left[g_{i, 1}\left(u_{i, 1}, x_{i, 1}\right)+\beta g_{i, 2}\left(x_{i, 2}, x_{i, 2}\right)\right]$,

s.t. $0<u_{i, 1} \leqslant x_{1}$,

where the water stock in the last period $x_{i, 2}$ is given by Eq. (4).

We note that the problem stated in Eqs. (4), (6) and (7) corresponds to a single period strategic form game given by the payoff function $\Gamma_{i, 1}\left(\vec{u}_{1}, \vec{x}_{1}\right)$ and the strategy set $u_{i, 1}$. We observe that the strategy set; $0<u_{i, 1} \leqslant x_{1}$, is nonempty, continuous, convex and compact (closed and bounded) and that the payoff function is continuous and jointly concave in the players' strategies as implied by Proposition 1. Then, from Theorem 1 in Dasgubta and Maskin [5], we have the following result.

Proposition 2 (Existence of Nash equilibrium). The $n$-player game which corresponds to the decentralized problem in the strip configuration has (at least one) Nash equilibrium.

A Nash equilibrium corresponds to the simultaneous solution of $n$ constrained optimization problems given above. If the Nash equilibrium occurs such that no user depletes his initial water stock in the first period $\left(u_{i, 1}^{*}<x_{1}, \forall i\right)$, then we have the unconstrained solution. Although it cannot be guaranteed in general, this result appears to us as the most common, real-life solution. Moreover, we are able to obtain further structural results and elegant solutions for the unconstrained optimization problem, which we shall present shortly. For completeness, we need also to consider the case of constrained solutions where $u_{i, 1}^{*}=x_{1}$. To this end, we construct the Lagrange function $L\left(u_{i, 1}, \delta_{i}\right)=\Gamma_{i, 1}\left(\vec{u}_{1}, \vec{x}_{1}\right)+\delta_{i}\left(x_{1}-u_{i, 1}\right)$, where $\delta_{i} \geqslant 0$ is the Lagrange multiplier corresponding to the constraint $u_{i, 1} \leqslant x_{1}$. Let $\vec{u}_{1}^{*}=\left(u_{1,1}^{*}, \ldots, u_{n, 1}^{*}\right)^{T}$ be the vector of optimal water usage in period $1, \vec{\delta}^{*}=\left(\delta_{1}^{*}, \ldots, \delta_{n}^{*}\right)^{T}$ be the optimal vector of the Lagrange multipliers, $\vec{x}_{1}=\left(x_{1}, \ldots, x_{1}\right)^{T}$ be an $n \times 1$ vector of initial water stock in period 1 and $\overrightarrow{0}=(0, \ldots, 0)^{T}$ be an $n \times 1$ zero vector. Then, as shown in the Online Supplement for Proposition 3, the Karush-Kuhn-

Tucker (KKT) conditions of the Lagrange function give the following.

$A \vec{u}_{1}^{*}-\vec{\delta}^{* T}=W$

$\vec{\delta}^{* T}\left(\vec{x}_{1}-\vec{u}_{1}^{*}\right)=0$,

$\vec{u}_{1}^{*} \leqslant \vec{x}_{1}$,

$\vec{\delta}^{*} \geqslant \overrightarrow{0}$,

$\begin{array}{cccccc}\text { where } & A_{n \times n}= & \left(\begin{array}{cccccc}\gamma_{1} & \sigma_{1} & 0 & 0 & \cdots & 0 \\ \epsilon_{1} & \gamma_{2} & \sigma_{2} & 0 & \cdots & 0 \\ 0 & \epsilon_{2} & \gamma_{3} & \sigma_{3} & \cdots & 0 \\ \vdots & \vdots & \vdots & \vdots & \vdots & \vdots \\ 0 & 0 & \cdots & \epsilon_{n-2} & \gamma_{n-1} & \sigma_{n-1} \\ 0 & 0 & \cdots & 0 & \epsilon_{n-1} & \gamma_{n}\end{array}\right), \quad W_{n \times 1}= \\ \left(\begin{array}{lllllll}\lambda_{1} & \lambda_{2} & \cdots & \lambda_{n-1} & \lambda_{n}\end{array}\right)^{T} \text { and } & & & \end{array}$ $\gamma_{i}= \begin{cases}\beta(1-\alpha)^{2}\left(c_{i, 2}-\rho_{i, 2} b_{i, 2}\right)-\left(\rho_{i, 1} b_{i, 1}+c_{i, 1}\right), & i=1, n, \\ \beta(1-2 \alpha)^{2}\left(c_{i, 2}-\rho_{i, 2} b_{i, 2}\right)-\left(\rho_{i, 1} b_{i, 1}+c_{i, 1}\right), & \text { o.w. }\end{cases}$

$\lambda_{i}=\left\{\begin{array}{l}\beta(1-\alpha)\left[\rho_{i, 2}\left(a_{i, 2}-b_{i, 2} x_{1}\right)+\left(c_{i, 2}-\rho_{i, 2} b_{i, 2}\right) w_{1}\right]-\rho_{i, 1} a_{i, 1}, \\ \quad i=1, n, \\ \beta(1-2 \alpha)\left[\rho_{i, 2}\left(a_{i, 2}-b_{i, 2} x_{1}\right)+\left(c_{i, 2}-\rho_{i, 2} b_{i, 2}\right) w_{1}\right]-\rho_{i, 1} a_{i, 1}, \\ \quad \text { o.w. }\end{array}\right.$

$\sigma_{i}=\left\{\begin{array}{ll}\beta \alpha(1-\alpha)\left(c_{i, 2}-\rho_{i, 2} b_{i, 2}\right), & i=1, \\ \beta \alpha(1-2 \alpha)\left(c_{i, 2}-\rho_{i, 2} b_{i, 2}\right), & \text { o.w. }\end{array}\right.$ and $\epsilon_{i}= \begin{cases}\beta \alpha(1-\alpha)\left(c_{n, 2}-\rho_{n, 2} b_{n, 2}\right), & i=n-1, \\ \beta \alpha(1-2 \alpha)\left(c_{i, 2}-\rho_{i, 2} b_{i, 2}\right), & \text { o.w. }\end{cases}$

Proposition 1 implies that the Hessian of $\Gamma_{i, 1}$ is negative semi-definite, and, hence, the two-period decentralized problem is a concave quadratic program. Therefore, the KKT conditions in (8)-(11) are, in fact, sufficient for $\vec{u}_{1}^{*}$ to be a global optimal solution as mentioned in Nocedal and Wright [12]. Several classes of algorithms have been used for solving concave quadratic problems that contain both inequality and equality constraints. Active-set methods have for long been used and are proved to be effective for small- and medium-sized problems. However, a special type of active-set methods called the gradient projection method has recently been shown most effective for solving concave quadratic problems having only upper and lower bounds as constraints on the decision variables, as discussed in Nocedal and Wright [12]. Hence, any one of these methods may be employed for solving the KKT conditions above since we have only the upper bound on decision variables. Clearly, if $\delta_{i}^{*}=0$ in the solution for the above Lagrange function for all $i$, then, the optimal solution is the unconstrained solution (global maximizer of $\Gamma_{i, 1}\left(\vec{u}_{1}, \vec{x}_{1}\right)$ in $\left.\mathfrak{R}^{+}\right)$, which we consider next. First, we establish the uniqueness of the unconstrained optimal solution.

Proposition 3. Uniqueness of the global maximizer and opt imal ity).

(i) The global maximizer of $\Gamma_{i, 1}\left(\vec{u}_{1}, \vec{x}_{1}\right)$ is unique and given by $u_{1,1}^{* *}=\frac{\tau_{1}}{\kappa}, u_{2,1}^{* *}=\frac{\tau_{2}}{\kappa}$ and $u_{k+2,1}^{* *}=\hat{\lambda}_{k+2}+\hat{e}_{(k+2,1)} u_{1,1}^{* *}+\hat{e}_{(k+2,2)} u_{2,1}^{* *}$, for $k=1, \ldots, n-2$, where $\tau_{1}=\lambda_{1}\left[\sum_{j=0}^{1} e_{(n, n-j)} \hat{e}_{(n-j, 2)}\right]-\sigma_{1}\left[\lambda_{n}-\right.$ $\left.\sum_{j=0}^{1} e_{(n, n-j)} \hat{\lambda}_{n-j}\right], \tau_{2}=\gamma_{1}\left[\lambda_{n}-\sum_{j=0}^{1} e_{(n, n-j)} \hat{\lambda}_{n-j}\right]-\lambda_{1}\left[\sum_{j=0}^{1} e_{(n, n-j)} \times\right.$ $\left.\hat{e}_{(n-j, 1)}\right], \kappa=\gamma_{1}\left[\sum_{j=0}^{1} e_{(n, n-j)} \hat{e}_{(n-j, 2)}\right]-\sigma_{1}\left[\sum_{j=0}^{1} e_{(n, n-j)} \hat{e}_{(n-j, 1)}\right], \hat{\lambda}_{k+2}=$ $\left[\lambda_{k+1}-\sum_{j=1}^{2} e_{(k+1, k+2-j)} \hat{\lambda}_{k+2-j}\right] /\left[e_{(k+1, k+2)}\right], \hat{e}_{(k+2, m)}=-\left[\sum_{j=1}^{2} \times\right.$ $\left.\boldsymbol{e}_{(k+1, k+2-j)} \hat{\boldsymbol{e}}_{(k+2-j, m)}\right] /\left[\boldsymbol{e}_{(k+1, k+2)}\right]$, for $m=1,2 ; \hat{\lambda}_{1}=\hat{\lambda}_{2}=0, \hat{e}_{(1,1)}=$ $\hat{e}_{(2,2)}=1, \hat{e}_{(1,2)}=\hat{e}_{(2,1)}=0$ and $e_{(m, i)}=\hat{e}_{(m, 1)}=\hat{e}_{(m, 2)}=0$, for $\{i, m\}<1$ and $\{i, m\}>n ;$ for $i=1, \ldots, n, e_{(i, i)}=\gamma_{i}$ and $e_{(i, j)}=\left\{\begin{array}{ll}\sigma_{i}, & (i, j)=(i, i+1), i=1, \ldots, n-1 \\ \epsilon_{i}, & (i, j)=(i, i-1), i=2, \ldots, n \\ 0, & \text { o.w. }\end{array}\right.$.

(ii) If $0 \leqslant u_{i, 1}^{* *} \leqslant x_{1}$, for all $i$, then $u_{i, 1}^{* *}$, given above, is the optimal solution for the decentralized problem.

When all users are identical, we have $g_{i, t}=g_{t}$ for all $i$. In the sequel, in $A$ and $W$, we have

$\gamma_{i}=\left\{\begin{array}{ll}\gamma, i=1, n \\ \epsilon, & \text { o.w. }\end{array}, \quad \sigma_{i}=\left\{\begin{array}{ll}\omega, & i=1 \\ \sigma, & \text { o.w. }\end{array}\right.\right.$,

$\epsilon_{i}=\left\{\begin{array}{ll}\omega, & i=n-1 \\ \sigma, & 0 . \mathrm{w} .\end{array}\right.$ and $\lambda_{i}=\left\{\begin{array}{ll}\eta, & i=1, n \\ \lambda, & 0 . \mathrm{w} .\end{array}\right.$,

where $\gamma=\beta(1-\alpha)^{2}\left(c_{2}-\rho_{2} b_{2}\right)-\left(\rho_{1} b_{1}+c_{1}\right), \quad \epsilon=\beta(1-2 \alpha)^{2}\left(c_{2}-\right.$ $\left.\rho_{2} b_{2}\right)-\left(\rho_{1} b_{1}+c_{1}\right), \quad \omega=\beta \alpha(1-\alpha)\left(c_{2}-\rho_{2} b_{2}\right), \quad \sigma=\beta \alpha(1-2 \alpha)\left(c_{2}-\right.$ $\left.\rho_{2} b_{2}\right), \quad \eta=\beta(1-\alpha)\left[\rho_{2}\left(a_{2}-b_{2} x_{1}\right)+\left(c_{2}+\rho_{2} b_{2}\right) w_{1}\right]-\rho_{1} a_{1}$ and 
$\lambda=\beta(1-2 \alpha)\left[\rho_{2}\left(a_{2}-b_{2} x_{1}\right)+\left(c_{2}-\rho_{2} b_{2}\right) w_{1}\right]-\rho_{1} a_{1}$. In this case, the system can be characterized through difference equations with location index as the argument (see Elaydi [6]); hence, we have a closed form result for the optimal solution to the unconstrained problem.

Corollary 2 (Unique global maximizer for identical users). For $n$-identical users on a strip, let $k=n / 2$ if $n$ is even and $(n+1) / 2$ otherwise. Then, the system $A \vec{u}_{1}^{* *}=W$ has a unique solution given by $u_{i, 1}^{* *}=u_{n-i+1,1}^{* *}=h_{0}+h_{1}\left(r_{1}\right)^{i}+h_{2}\left(r_{2}\right)^{i}, i=1, \ldots, k$, where $h_{0}=\lambda /$ $(2 \sigma+\epsilon), r_{1}=\left(-\epsilon-\sqrt{\epsilon^{2}-4 \sigma^{2}}\right) / 2 \sigma, r_{2}=\left(-\epsilon+\sqrt{\epsilon^{2}-4 \sigma^{2}}\right) / 2 \sigma$ and for $k=n / 2$,

$h_{1}=\frac{\left[\eta-\left(\frac{\gamma+\omega}{2 \sigma+\epsilon}\right) \lambda\right]}{\left[\gamma r_{1}+\omega\left(r_{1}\right)^{2}\right]-\left[\gamma r_{2}+\omega\left(r_{2}\right)^{2}\right]\left[\frac{\sigma+(\sigma+\epsilon) r_{1}}{\sigma+(\sigma+\epsilon) r_{2}}\right]\left(\frac{r_{1}}{r_{2}}\right)^{(k-1)}} ;$

$h_{2}=-h_{1}\left[\frac{\sigma+(\sigma+\epsilon) r_{1}}{\sigma+(\sigma+\epsilon) r_{2}}\right]\left(\frac{r_{1}}{r_{2}}\right)^{(k-1)}$

and for $k=(n+1) / 2$,

$h_{1}=\frac{\left[\eta-\left(\frac{\gamma+\omega}{2 \sigma+\epsilon}\right) \lambda\right]}{\left[\gamma r_{1}+\omega\left(r_{1}\right)^{2}\right]-\left[\gamma r_{2}+\omega\left(r_{2}\right)^{2}\right]\left[\frac{2 \sigma+\epsilon r_{1}}{2 \sigma+\epsilon r_{2}}\right]\left(\frac{r_{1}}{r_{2}}\right)^{(k-1)}} ;$

$h_{2}=-h_{1}\left[\frac{2 \sigma+\epsilon r_{1}}{2 \sigma+\epsilon r_{2}}\right]\left(\frac{r_{1}}{r_{2}}\right)^{(k-1)}$.

Remarks. (1). We note Saak and Peterson [14] find that the Nash equilibrium for $n=2$ gives water usages for both users that are symmetric, unique and dependent on lateral flow coefficient $\alpha$. Corollary 2 also implies that the unconstrained optimal solution is symmetric around the mid-point (s) of the strip and generalizes their findings to the case where $n>2$. (2). Since $\epsilon$ and $\sigma$ are negative, we have $r_{1}, r_{2}<0$ and $r_{1}>r_{2}$. This implies that the unconstrained optimal solution has a fluctuating structure across the users from the extremes toward the center. Thus, for the unconstrained optimal solution, we have established theoretically Saak and Peterson's [14] conjecture (p. $226)$ that water usage would not be monotone for multiple users $(n>2)$ even when they are all identical. We think that this has significance for policy makers in the design of payment schemes (cost structures) for underground water usage for multiple users $(n>2)$. In our numerical study, we have observed that, typically, the second most extreme users at both ends of the strip have the highest water consumption in the unconstrained solutions. If this observation always holds, then it may be possible to obtain the cost-revenue parameter space so that the Nash equilibrium always occurs as the unconstrained optimal. (3). In the above formulation of the problem, we have assumed that users have complete information about other players' parameters and the hydrological properties of the aquifer expressed through $\alpha$. An interesting variant of the problem analyzed by Saak and Peterson [14] for $n=2$ is the case where users have incomplete information about $\alpha$ considered to be a random variable. In the case of identical users, it turns out that, also for $n>2$, the problem can be stated as the expected total discounted profits and all of the results provided so far involving $\alpha$ would still hold in the expectation sense; that is, $E[\alpha]$ in place of $\alpha, E\left[(1-\alpha)^{2}\right]$ in place of $(1-\alpha)^{2}$ etc. For non-identical users, incorporation of asymmetry of information seems not so straightforward. We examine further properties of the optimal solutions in our numerical study.

\subsection{The centralized problem}

In this problem, we envision a central decision maker (social planner in the public policy parlance) aiming at determining the optimal water usage for each user so that the total joint discounted profit of all users throughout the planning horizon of two periods is maximized. The problem can be formally stated as a dynamic programming problem as follows. For $t=1,2$, and $i=1, \ldots, n$,

$$
\begin{aligned}
\widetilde{\Gamma}_{t}^{*}\left(\vec{u}_{t}, \vec{x}_{t}\right) & =\max _{u_{i, t}, \ldots, u_{n, t}} \widetilde{\Gamma}_{t}\left(\vec{u}_{t}, \vec{x}_{t}\right) \\
& =\max _{u_{i, t}, \ldots, u_{n, t}}\left\{\left\{\sum_{i=1}^{n} g_{i, t}\left(u_{i, t}, x_{i, t}\right)\right\}+\beta_{t} \widetilde{\Gamma}_{t+1}^{*}\left(\vec{u}_{t+1}, \vec{x}_{t+1}\right)\right\}
\end{aligned}
$$

s.t. (4) and (7)

where $\widetilde{\Gamma}_{t}\left(\vec{u}_{t}, \vec{x}_{t}\right)$ is the joint profit-to-go function from period $t$ until the end of the problem horizon. All of the other conventions and notations of the decentralized problem are retained. Since $\widetilde{\Gamma}_{t}\left(\vec{u}_{t}, \vec{x}_{t}\right)$ is a positive linear combination of individual discounted profit-to-go functions in the decentralized problem, we immediately have the following.

Corollary 3. Myopic optimality, positivity, continuity, concavity

(i) The myopically optimal water usage in period t is to deplete all stock $\left.g_{i, t}^{*}\left(u_{i, t}, x_{i, t}\right)=g_{i, t}\left(x_{i, t}, x_{i, t}\right)\right]$.

(ii) For a given $\vec{x}_{1}, \widetilde{\Gamma}_{1}\left(\vec{u}_{1}, \vec{x}_{1}\right)$ is strictly increasing in $u_{i, 1}$ at $u_{i, 1}=0$ if $\rho_{i, 1} a_{i, 1} \geqslant \beta\left(\rho_{i, 2} a_{i, 2}+c_{i, 2} w_{1}\right)$, for all $i$.

(iii) For a given $\vec{x}_{1}, \widetilde{\Gamma}_{1}\left(\vec{u}_{1}, \vec{x}_{1}\right)$ is continuous and jointly concave in $\vec{u}_{1}$ if and only if $c_{i, 2} \leqslant \rho_{i, 2} b_{i, 2}$, for all $i$.

The above imply that the centralized problem also reduces to an equivalent single period concave quadratic optimization problem subject to the constraint set $0<u_{i, 1} \leqslant x_{1}$, for all $i$. Constructing the Lagrange function for this problem $L\left(u_{i, 1}, \delta_{i}\right)=\widetilde{\Gamma}_{i}\left(\vec{u}_{t}, \vec{x}_{t}\right)+$ $\delta_{i}\left(x_{1}-u_{i, 1}\right)$, the KKT conditions result in $\widetilde{A} \vec{u}_{1}^{*}-\vec{\delta}^{* T}=\widetilde{W}$, together with (9)-(11). The unconstrained solution of the centralized problem corresponding to the general case of non-identical users is given in the following result.

Proposition 4. Uniqueness of the global maximizer and optimality).

(i) The global maximizer of $\widetilde{\Gamma}_{1}\left(\vec{u}_{1}, \vec{x}_{1}\right)$ is unique and given by $u_{1,1}^{* *}=\frac{\tilde{\lambda}_{1}}{\tilde{\omega}}, u_{2,1}^{* *}=\frac{\tilde{\lambda}_{2}}{\tilde{\omega}}$ and $u_{k+2,1}^{* *}=\hat{\theta}_{k+2}+\hat{e}_{(k+2,1)} u_{1,1}^{* *}+\hat{e}_{(k+2,2)} u_{2,1}^{* *}$, for $k=1, \ldots, n-2$, where

$$
\begin{aligned}
& \tilde{\lambda}_{1}= \sum_{j=0}^{2} e_{(n, n-j)} \hat{e}_{(n-j, 2)}\left[\theta_{n-1}-\sum_{j=0}^{3} e_{(n-1, n-j)} \hat{\theta}_{n-j}\right] \\
&-\sum_{j=0}^{3} e_{(n-1, n-j)} \hat{e}_{(n-j, 2)}\left[\theta_{n}-\sum_{j=0}^{2} e_{(n, n-j)} \hat{\theta}_{n-j}\right] \\
& \tilde{\lambda}_{2}= \sum_{j=0}^{3} e_{(n-1, n-j)} \hat{e}_{(n-j, 1)}\left[\theta_{n}-\sum_{j=0}^{2} e_{(n, n-j)} \hat{\theta}_{n-j}\right] \\
&-\sum_{j=0}^{2} e_{(n, n-j)} \hat{e}_{(n-j, 1)}\left[\theta_{n-1}-\sum_{j=0}^{3} e_{(n-1, n-j)} \hat{\theta}_{n-j}\right] \\
& \tilde{\omega}_{=} \sum_{j=0}^{3} e_{(n-1, n-j)} \hat{e}_{(n-j, 1)} \sum_{j=0}^{2} e_{(n, n-j)} \hat{e}_{(n-j, 2)} \\
&-\sum_{j=0}^{3} e_{(n-1, n-j)} \hat{e}_{(n-j, 2)} \sum_{j=0}^{2} e_{(n, n-j)} \hat{e}_{(n-j, 1)}, \\
& \hat{\theta}_{k+2}= {\left[\theta_{k}-\sum_{j=1}^{4} e_{(k, k+2-j)} \hat{\theta}_{k+2-j}\right] /\left[e_{(k, k+2)}\right], } \\
& \hat{e}_{(k+2, m)}=-\left[\sum_{j=1}^{4} e_{(k, k+2-j)} \hat{e}_{(k+2-j, m)}\right] /\left[e_{(k, k+2)}\right]
\end{aligned}
$$


for $m=1,2 ; \quad \hat{\theta}_{1}=\hat{\theta}_{2}=0, \hat{e}_{(1,1)}=\hat{e}_{(2,2)}=1, \hat{e}_{(1,2)}=\hat{e}_{(2,1)}=0$ and $e_{(m, i)}=\hat{e}_{(m, 1)}=\hat{e}_{(m, 2)}=0$, for $\{m, i\}<1$ and $\{m, t i\}>n ; e_{(i, i)} ;=\left(\rho_{i, 1-}\right.$ $\left.\rho_{i, 1} b_{i, 1}+c_{i, 1}\right)+\beta(1-\alpha)^{2}\left(\rho_{i, 2} b_{i, 2}-c_{i, 2}\right)+\beta \alpha^{2}\left(\rho_{j, 2} b_{j, 2}-c_{j, 2}\right), \quad(i, j) \in$ $\{(1,2),(n, n-1)\}$,

$$
\begin{aligned}
e_{(i, i)}= & \left(\rho_{i, 1} b_{i, 1}+c_{i, 1}\right)+\beta(1-2 \alpha)^{2}\left(\rho_{i, 2} b_{i, 2}-c_{i, 2}\right) \\
+ & \beta \alpha^{2}\left[\left(\rho_{i-1,2} b_{i-1,2}-c_{i-1,2}\right)+\left(\rho_{i+1,2} b_{i+1,2}-c_{i+1,2}\right)\right], \\
& i=2, \ldots, n-1, \\
e_{(i, m)}= & \beta \alpha^{2}\left(\rho_{j, 2} b_{j, 2}-c_{j, 2}\right),(i, j, m) \in\{(1,2,3), \\
& (n, n-1, n-2),(2,3,4),(n-1, n-2, n-3), \\
& (k, k-1, k-2),(k, k+1, k+2)\} \\
e_{(i, j)}= & \beta \alpha(1-\alpha)\left(\rho_{i, 2} b_{i, 2}-c_{i, 2}\right)+\beta \alpha(1-2 \alpha)\left(\rho_{j, 2} b_{j, 2}-c_{j, 2}\right), \\
& (i, j) \in\{(1,2),(n, n-1)\} \\
e_{(i, j)}= & \beta \alpha(1-2 \alpha)\left(\rho_{i, 2} b_{i, 2}-c_{i, 2}\right)+\beta \alpha(1-\alpha) \\
& \times\left(\rho_{j, 2} b_{j, 2}-c_{j, 2}\right),(i, j) \in\{(2,1),(n-1, n)\}, \\
e_{(i, j)}= & \beta \alpha(1-2 \alpha)\left[\left(\rho_{i, 2} b_{i, 2}-c_{i, 2}\right)+\left(\rho_{j, 2} b_{j, 2}-c_{j, 2}\right)\right], \\
& (i, j) \in\{(2,3),(n-1, n-2),(k, k-1),(k, k+1)\}, \\
e_{(i, j)}= & 0, \quad \text { elsewhere; } \\
\theta_{i}= & \rho_{i, 1} a_{i, 1}-\beta(1-\alpha)\left(\rho_{i, 2} a_{i, 2}+c_{i, 2} w_{1}\right)-\beta \alpha\left(\rho_{j, 2} a_{j, 2}+c_{j, 2} w_{1}\right) \\
+ & \beta(1-\alpha) \rho_{i, 2} b_{i, 2}\left(x_{1}+w_{1}\right)+\beta \alpha \rho_{j, 2} b_{j, 2}\left(x_{1}+w_{1}\right), \\
& (i, j) \in\{(1,2),(n, n-1)\} \text { and } \\
\theta_{i}= & \rho_{i, 1} a_{i, 1}-\beta(1-2 \alpha)\left(\rho_{i, 2} a_{i, 2}+c_{i, 2} w_{1}\right)-\beta \alpha\left[\left(\rho_{i-1,2} a_{i-1,2}+c_{i-1,2} w_{1}\right)\right. \\
+ & \left.\left(\rho_{i+1,2} a_{i+1,2}+c_{i+1,2} w_{1}\right)\right]+\beta(1-2 \alpha) \rho_{i, 2} b_{i, 2}\left(x_{1}+w_{1}\right) \\
+ & \beta \alpha\left[\rho_{i-1,2} b_{i-1,2}\left(x_{1}+w_{1}\right)+\rho_{i+1,2} b_{i+1,2}\left(x_{1}+w_{1}\right)\right], \\
& i=2, \ldots, n-1 .
\end{aligned}
$$

(ii) If $0 \leqslant u_{i, 1}^{* *} \leqslant x_{1}$, for all $i$, then $u_{i, 1}^{* *}$, given above, is the optimal solution for the centralized problem.

For identical users, we establish that the global maximizer of $\widetilde{\Gamma}_{1}$ in $\mathfrak{R}^{+}$is unique, independent of the hydrological properties of the aquifer $(\alpha)$ and it is the same for all users unlike the decentralized solution. Furthermore, the unconstrained solution is the optimal for the centralized problem for certain cost and revenue parameter values. We state this result below.

Corollary 4. Uniqueness of the global maximizer and optimality for identical users).

(i) Suppose that users are identical and $c_{2} \leqslant \rho_{2} b_{2}$. Then, the global maximizer of $\widetilde{\Gamma}_{1}\left(\vec{u}_{1}, \vec{x}_{1}\right)$ is unique and given by

$$
\begin{aligned}
u_{i, 1}^{* *}= & u^{* *} \\
= & {\left[\rho_{1} a_{1}-\beta\left(\rho_{2} a_{2}+c_{2} w_{1}\right)+\beta \rho_{2} b_{2}\left(x_{1}+w_{1}\right)\right] /\left[\left(\rho_{1} b_{1}+c_{1}\right)\right.} \\
& \left.+\beta\left(\rho_{2} b_{2}-c_{2}\right)\right], \quad \forall i .
\end{aligned}
$$

(ii) If $0 \leqslant u_{i, 1}^{* *} \leqslant x_{1}$, for all $i$, then the optimal solution for the centralized problem is given by $u^{* *}$ above.

Remarks (1). Saak and Peterson [14] have shown for $n=2$ that the optimal solution is independent of the characteristics of the aquifer expressed through $\alpha$. Hence, Corollary 4 generalizes this finding. However, Saak and Peterson [14] make an implicit assumption that the Nash equilibrium will be the unconstrained solution throughout their analysis. In our result, we establish the conditions for the optimality of the global maximizer to be within the constraint set. (2). The conditions for the optimal solution above imply that, under the cost-revenue assumptions of Saak and Peterson [14], the centralized problem results in an optimal usage which does not deplete the initial stock when $0.5 \leqslant \beta \leqslant 1$ - giving a realistic hurdle rate between $0 \%$ and $100 \%$ per period. Hence, we think that the above optimal result would be observed in most realistic cases. (3). From a policy maker's perspective, it is important to know if the centralized solution can be achieved in the decentralized game- theoretic setting through a pricing mechanism. Under the stated condition above, the optimal solution dictates the same usage for all users. However, in the decentralized solution for the unconstrained case, we established that water usage fluctuates from the ends toward the midpoint (s) of the strip. As these constitute instances of counter examples, we establish by contradiction the following.Corollary 5. No coordination). In a strip configuration with $n$ identical users, for $\left(\rho_{t} b_{t}+c_{t}\right) x_{0}<\rho_{t} a_{t}<\left(2 \rho_{t} b_{t}+c_{t}\right) x_{0}$, there does not exist a periodic unit pumping cost $c_{t}$ that equates the Nash equilibrium with the centralized optimal solution, for all $t$.

We present further observations about the optimal solution in our numerical section.

\section{Ring configuration}

In this section, we consider the setting where all $n$ users are connected to each other in a ring or circular configuration. By definition of a ring, we have $n>2$. Unlike the strip configuration examined above, there are no locational extremes (ends) and each user has exactly two neighbors. Hence, the lateral flows in the aquifer makes all users communicate with each other; and, one particular user's water consumption affects all users in the system either directly or indirectly. The more even nature of the structure brings a similar evenness to the solution as well, as shall be discussed below. Users are numbered in a clockwise fashion where each user has lateral flow from one preceding and one succeeding adjacent user in the ring. In this configuration, we consider below the decentralized and centralized decision making environments.

\subsection{The decentralized problem}

The decentralized problem for the ring configuration is similar to that for the strip configuration except that the recursive relation between water stocks over time is different owing to the non-existence of any ends of a ring. For $t=1,2$, the decentralized problem for user $i, i=1, \ldots, n$, is formally stated as a dynamic program given by

$$
\begin{aligned}
& \Gamma_{i, t}^{*}\left(\vec{u}_{t}, \vec{x}_{t}\right)= \max _{u_{i, t}} \Gamma_{i, t}\left(\vec{u}_{t}, \vec{x}_{t}\right) \\
&= \max _{u_{i, t}}\left[g_{i, t}\left(u_{i, t}, x_{i, t}\right)+\beta_{i, t} \Gamma_{i, t+1}^{*}\left(\vec{u}_{t+1}, \vec{x}_{t+1}\right)\right], \\
& \text { s.t. } x_{i, t+1}=\left\{\begin{array}{l}
x_{i, t}+w_{i, t}-(1-2 \alpha) u_{i, t}-\alpha\left(u_{j, t}+u_{m, t}\right), \\
(i, j, m) \in\{(1, n, 2),(n, n-1,1)\}, \\
x_{i, t}+w_{i, t}-(1-2 \alpha) u_{i, t}-\alpha\left(u_{i-1, t}+u_{i+1, t}\right), \\
i=2, \ldots, n-1,
\end{array}\right.
\end{aligned}
$$

$0 \leqslant u_{i, t} \leqslant x_{i, t}$

In the above, we retain the previous notations. Note that Eq. (14) describes the recursive temporal relationship among the water stocks of the users under Darcy's Law; unlike the strip, the ring configuration allows for each user to communicate with its immediate neighbors. As before, we have the same $\alpha$ for all users and all $t$; $\beta_{i, t}=\beta$ with $0 \leqslant \beta \leqslant 1$; we set $x_{i, 1}=x_{1}, w_{i, 1}=w_{1}$ and $\Gamma_{3}^{*}\left(\vec{u}_{3}, \vec{x}_{3}\right) \equiv 0$ for all $\vec{x}_{3}, \vec{u}_{3}$ and for $i=1, \ldots, n$. (We later relax the condition on $\left.\Gamma_{3}^{*}\left(\vec{u}_{3}, \vec{x}_{3}\right)\right)$. The properties of the within period profit function in Corollary 1 also imply that the decentralized problem in the ring configuration can be written as a single period problem, and that its objective function is also a well-behaving function as stated in the following result. 
Proposition 5. Positivity, continuity, concavity

(i) $\Gamma_{i, 1}\left(\vec{u}_{1}, \vec{x}_{1}\right)\left(=\left[g_{i, 1}\left(u_{i, 1}, x_{i, 1}\right)+\beta g_{i, 2}\left(x_{i, 2}, x_{i, 2}\right)\right]\right)$ is strictly increasing in $u_{i, 1}$ at $u_{i, 1}=0$ if $\rho_{i, 1} a_{i, 1} \geqslant \beta\left(\rho_{i, 2} a_{i, 2}+c_{i, 2} w_{1}\right), i=1, \ldots, n$.

(ii) $\Gamma_{i, 1}\left(\vec{u}_{1}, \vec{x}_{1}\right)$ is continuous and jointly concave in $\vec{u}_{1}$ if and only if $c_{i, 2} \leqslant \rho_{i, 2} b_{i, 2}, i=1, \ldots, n$.

The proof methodology is identical to that for Proposition 1 and, hence, is omitted. Proposition 5 enables a tighter reformulation of the $n$ - user problem given by Eq. (6) as the objective function subject to Eq. (7) where the water stock in the last period $x_{i, 2}$ is given by Eq. (14). As the properties of the problem satisfy those of Theorem 1 in Dasgubta and Maskin [5], we have the existence of a Nash equilibrium as stated below.

Proposition 6. Existence of Nash equilibrium The $n$ - player game which corresponds to the decentralized problem in the ring configuration has (at least one) Nash equilibrium.

The Nash equilibrium corresponds to the simultaneous solution of $n$ constrained optimization problems with a single constraint $u_{i, 1} \leqslant x_{1}, i=1, \ldots, n$. As shown in the Online Supplement for Proposition 7 , the KKT conditions of the Lagrange function $L\left(u_{i, 1}, \delta_{i}\right)=\Gamma_{i}\left(\vec{u}_{1}, \vec{x}_{1}\right)+\delta_{i}\left(x_{1}-u_{i, 1}\right)$, together with (9)-(11), give $B \vec{u}_{1}^{*}-\vec{\delta}^{* T}=Z$, where

$B_{n \times n}=\left(\begin{array}{cccccc}\epsilon_{1} & \sigma_{1} & 0 & 0 & \cdots & \sigma_{1} \\ \sigma_{2} & \epsilon_{2} & \sigma_{2} & 0 & \cdots & 0 \\ 0 & \sigma_{3} & \epsilon_{3} & \sigma_{3} & \cdots & 0 \\ \vdots & \vdots & \vdots & \vdots & \vdots & \vdots \\ 0 & 0 & \cdots & \sigma_{n-1} & \epsilon_{n-1} & \sigma_{n-1} \\ \sigma_{n} & 0 & \cdots & 0 & \sigma_{n} & \epsilon_{n}\end{array}\right), Z_{n \times 1}=\left(\lambda_{1} \lambda_{2} \cdots \lambda_{n-1} \lambda_{n}\right)^{T}$

and

$$
\begin{aligned}
\epsilon_{i} & =\beta(1-2 \alpha)^{2}\left(c_{i, 2}-\rho_{i, 2} b_{i, 2}\right)-\left(\rho_{i, 1} b_{i, 1}+c_{i, 1}\right), \sigma_{i} \\
& =\beta \alpha(1-2 \alpha)\left(c_{i, 2}-\rho_{i, 2} b_{i, 2}\right) \quad \text { and } \lambda_{i} \\
& =\beta(1-2 \alpha)\left[\rho_{i, 2}\left(a_{i, 2}-b_{i, 2} x_{1}\right)+\left(c_{i, 2}-\rho_{i, 2} b_{i, 2}\right) w_{1}\right]-\rho_{i, 1} a_{i, 1} .
\end{aligned}
$$

Proposition 5 implies that the Hessian matrix of $\Gamma_{i, 1}$ is negative semi-definite, and, hence, the problem is a concave quadratic program. Therefore, the KKT conditions above are, again, sufficient for $\vec{u}_{1}^{*}$ to be a global optimal solution; and, the above mentioned methods may be used to find it. Next, we focus on the unconstrained solution $\left(\delta_{i}^{*}=0, \forall i\right)$.

Proposition 7. Uniqueness of the global maximizer and optimality for non-identical users).

(i) Suppose that users are non-identical. Then, the global maximizer of $\Gamma_{i, 1}\left(\vec{u}_{1}, \vec{x}_{1}\right)$ is unique and given by $u_{1,1}^{* *}=\frac{\tilde{\tau}_{1}}{\tilde{\kappa}}, u_{2,1}^{* *}=\frac{\tilde{\tau}_{2}}{\tilde{\kappa}}$ and $u_{k+2,1}^{* *}=\hat{\lambda}_{k+2}+\hat{e}_{(k+2,1)} u_{1,1}^{* *}+\hat{e}_{(k+2,2)} u_{2,1}^{* *}$, for $k=1, \ldots, n-2$, where

$$
\begin{aligned}
\tilde{\tau}_{1} & =\left[\lambda_{1}-\sigma_{1} \hat{\lambda}_{n}\right]\left[\sum_{j=0}^{1} e_{(n, n-j)} \hat{e}_{(n-j, 2)}\right] \\
& -\left[\sigma_{1}+\sigma_{1} \hat{e}_{(n, 2)}\right]\left[\lambda_{n}-\sum_{j=0}^{1} e_{(n, n-j)} \hat{\lambda}_{n-j}\right], \\
\tilde{\tau}_{2} & =\left[\epsilon_{1}+\sigma_{1} \hat{e}_{(n, 1)}\right]\left[\lambda_{n}-\sum_{j=0}^{1} e_{(n, n-j)} \hat{\lambda}_{n-j}\right] \\
& -\left[\lambda_{1}-\sigma_{1} \hat{\lambda}_{n}\right]\left[\sigma_{n}+\sum_{j=0}^{1} e_{(n, n-j)} \hat{e}_{(n-j, 1)}\right],
\end{aligned}
$$

$$
\begin{aligned}
\tilde{\kappa}= & {\left[\epsilon_{1}+\sigma_{1} \hat{e}_{(n, 1)}\right]\left[\sum_{j=0}^{1} e_{(n, n-j)} \hat{e}_{(n-j, 2)}\right] } \\
& -\left[\sigma_{1}+\sigma_{1} \hat{e}_{(n, 2)}\right]\left[\sigma_{n}+\sum_{j=0}^{1} e_{(n, n-j)} \hat{e}_{(n-j, 1)}\right], \hat{\lambda}_{k+2}
\end{aligned}
$$

and $\hat{e}_{(k+2, m)}$ are as defined before in Proposition 3. In addition, we have, for $i=1, \ldots, n, e_{(i, i)}=\epsilon_{i}$ and

$$
e_{(i . j)}=\left\{\begin{array}{lll}
\sigma_{i}, & (i, j) \in\{(i, i+1),(1, n)\}, & i=1, \ldots, n-1, \\
\sigma_{i}, & (i, j) \in\{(i, i-1),(n, 1)\}, & i=2, \ldots, n, \\
0, & \text { o.w. }
\end{array} .\right.
$$

(ii) If $0 \leqslant u_{i, 1}^{* *} \leqslant x_{1}$, for all $i$, then $u_{i, 1}^{* *}$, given above, is the optimal solution for the decentralized problem.

When all users are identical (i.e. $\epsilon_{i}=\epsilon, \sigma_{i}=\sigma$ and $\lambda_{i}=\lambda$, where $\epsilon$, $\sigma, \lambda<0)$, it is possible to obtain a compact expression for the Nash equilibrium.

Corollary 6. Unique Nash equilibrium for identical users). The $n$-player game corresponding to the decentralized problem in a ring configuration has a unique Nash equilibrium given by, for all $i$,

$$
u_{i, 1}^{*}= \begin{cases}\lambda /(2 \sigma+\epsilon), & \lambda>(2 \sigma+\epsilon) x_{1}, \\ x_{1}, & \text { o.w. }\end{cases}
$$

Remarks(1). In a ring configuration with identical users, all users consume the same amount from the aquifer in each period. So long as the cost-revenue structure is such that the condition $\lambda>$ $(2 \sigma+\epsilon) x_{1}$ is satisfied, the water stock is not depleted; otherwise, all users deplete the initial stock in the first period leaving nothing for the next period. We think that this observation may have significant implications for policy makers in setting the unit costs for underground water usage if decentralized decision making is to be employed. (2). Since users' optimal decisions are identical, it may be possible to convince the users either $(i)$ into a cooperative game rather than the competitive one they are playing, or (ii) into enforcing a centralized decision. In the next section, we take up this important issue of possible coordination through unit prices; that is, whether or not single price mechanisms exist through which the decentralized solution may converge to the centralized optimal decision. (3). Similar to the strip configuration, it is possible to construct the above game with imperfect information about the parameter $\alpha$ by replacing the expressions involving $\alpha$ with their expectation for identical users.

\subsection{The centralized problem}

Analogous to the strip configuration, the centralized problem for the ring configuration envisions that a social planner aims at determining the optimal underground water usage for each user so as to maximize the total discounted profit for the entire system stated in Eq. (12) subject to Eq. (15) where $x_{i, 2}$ is characterized by Eq. (14). Since the objective function of the optimization is a positive linear combination of the individual profit-to-go functions, we have the following result.

Corollary 7. Myopic optimality, positivity, continuity, concavity

(i) The myopically optimal water usage in period t is to deplete all stock $\left[g_{i, t}^{*}\left(u_{i, t}, x_{i, t}\right)=g_{i, t}\left(x_{i, t}, x_{i, t}\right)\right]$.

(ii) For a given $\vec{x}_{1}, \widetilde{\Gamma}_{1}\left(\vec{u}_{1}, \vec{x}_{1}\right)$ is strictly increasing in $u_{i, 1}$ at $u_{i, 1}=0$ if $\rho_{i, 1} a_{i, 1} \geqslant \beta\left(\rho_{i, 2} a_{i, 2}+c_{i, 2} w_{1}\right)$, for all $i$. 
(iii) For a given $\vec{x}_{1}, \widetilde{\Gamma}_{1}\left(\vec{u}_{1}, \vec{x}_{1}\right)$ is continuous and jointly concave in $\vec{u}_{1}$ if and only if $c_{i, 2} \leqslant \rho_{i, 2} b_{i, 2}$, for all $i$.

The above result once again implies that the centralized problem in the ring configuration reduces to an equivalent single period concave quadratic optimization problem subject to the initial constraint set $u_{i, 1} \leqslant x_{1}$ for all $i$. Constructing the Lagrange function for this problem in a similar fashion, we observe that the KKT conditions are given by $\widetilde{B} \vec{u}_{1}^{*}-\vec{\delta}^{* T}=\widetilde{W}$, together with (9)-(11). Similar to the strip configuration, the method of finding the unconstrained solution for the general case of non-identical users is given below.

Proposition 8. Uniqueness of the global maximizer and optimality for non-identical users).

(i) Suppose that users are non-identical. Then, the global maximizer of $\widetilde{\Gamma}_{1}\left(\vec{u}_{1}, \vec{x}_{1}\right)$ is unique and given by $u_{1,1}^{* *}=\frac{\tilde{\gamma}_{1}}{\tilde{\sigma}}, u_{2,1}^{* *}=\frac{\tilde{\gamma}_{2}}{\tilde{\sigma}}$, $u_{3,1}^{* *}=\frac{\tilde{\epsilon}_{2}-\tilde{d}_{2,1} u_{1,1}^{* *}-\tilde{d}_{2,2} u_{2,1}^{* *}}{\tilde{d}_{2,3}}, \quad u_{4,1}^{* *}=\frac{\epsilon_{1}-d_{1,1} u_{1,1}^{* *}-d_{1,2} u_{2,1}^{* *}-d_{1,3} u_{3,1}^{* *}}{d_{1,4}} a n d u_{k+2,1}^{* *}=$ $\hat{\phi}_{k+2}+\hat{e}_{(k+2,1)} u_{1,1}^{* *}+\hat{e}_{(k+2,2)} u_{2,1}^{* *}+\hat{e}_{(k+2,3)} u_{3,1}^{* *}+\hat{e}_{(k+2,4)} u_{4,1}^{* *}, \quad$ for $k=3, \ldots, n-2$, where

$$
\begin{aligned}
\tilde{\gamma}_{1}= & \left(\tilde{d}_{2,3} \tilde{\epsilon}_{3}-\tilde{d}_{3,3} \tilde{\epsilon}_{2}\right)\left(\tilde{d}_{4,2} \tilde{d}_{2,3}-\tilde{d}_{4,3} \tilde{d}_{2,2}\right) \\
& -\left(\tilde{d}_{2,3} \tilde{\epsilon}_{4}-\tilde{d}_{4,3} \tilde{\epsilon}_{2}\right)\left(\tilde{d}_{3,2} \tilde{d}_{2,3}-\tilde{d}_{3,3} \tilde{d}_{2,2}\right), \\
\tilde{\gamma}_{2}= & \left(\tilde{d}_{2,3} \tilde{\epsilon}_{4}-\tilde{d}_{4,3} \tilde{\epsilon}_{2}\right)\left(\tilde{d}_{3,1} \tilde{d}_{2,3}-\tilde{d}_{3,3} \tilde{d}_{2,1}\right) \\
& -\left(\tilde{d}_{2,3} \tilde{\epsilon}_{3}-\tilde{d}_{3,3} \tilde{\epsilon}_{2}\right)\left(\tilde{d}_{4,1} \tilde{d}_{2,3}-\tilde{d}_{4,3} \tilde{d}_{2,1}\right), \\
\tilde{\sigma}= & \left(\tilde{d}_{3,1} \tilde{d}_{2,3}-\tilde{d}_{3,3} \tilde{d}_{2,1}\right)\left(\tilde{d}_{4,2} \tilde{d}_{2,3}-\tilde{d}_{4,3} \tilde{d}_{2,2}\right) \\
& -\left(\tilde{d}_{3,2} \tilde{d}_{2,3}-\tilde{d}_{3,3} \tilde{d}_{2,2}\right)\left(\tilde{d}_{4,1} \tilde{d}_{2,3}-\tilde{d}_{4,3} \tilde{d}_{2,1}\right), \\
\tilde{\epsilon}_{i}= & d_{1, i} \epsilon_{i}-d_{i, 4} \epsilon_{1}, \tilde{d}_{i, j}=d_{1,4} d_{i, j}-d_{i, 4} d_{1, j}, \\
& \text { for } i=2,3,4 \text { and } j=1,2,3,
\end{aligned}
$$

$d_{1, m}=\left\{\begin{array}{ll}e_{(1, m)}+\sum_{j=0}^{1} e_{(1, n-j)} \hat{e}_{(n-j, m)}, & m=1,2,3 \\ \sum_{j=0}^{1} e_{(1, n-j)} \hat{e}_{(n-j, 4)}, & m=4\end{array}\right.$,

$d_{2, m}=e_{(2, m)}+e_{(2, n)} \hat{e}_{(n, m)}, \quad m=1,2,3,4$,

$d_{3, m}=\left\{\begin{array}{ll}e_{(n-1,1)}+\sum_{j=0}^{3} e_{(n-1, n-j)} \hat{e}_{(n-j, 1)}, & m=1 \\ \sum_{j=0}^{3} e_{(n-1, n-j)} \hat{e}_{(n-j, m)}, & m=2,3,4\end{array}\right.$,

$d_{4, m}= \begin{cases}e_{(n, m)}+\sum_{j=0}^{2} e_{(n, n-j)} \hat{e}_{(n-j, m)}, & m=1,2 \\ \sum_{j=0}^{2} e_{(n, n-j)} \hat{e}_{(n-j, m)}, & m=3,4\end{cases}$

$\epsilon_{1}=\phi_{1}-\sum_{j=0}^{1} e_{(1, n-j)} \hat{\phi}_{n-j}$,

$\epsilon_{2}=\phi_{2}-e_{(2, n)} \hat{\phi}_{n}$

$\epsilon_{3}=\phi_{n-1}-\sum_{j=0}^{3} e_{(n-1, n-j)} \hat{\phi}_{n-j}$,

$\epsilon_{4}=\phi_{n}-\sum_{j=0}^{3} e_{(n, n-j)} \hat{\phi}_{n-j}$, with $\hat{\phi}_{k+2}=\left[\phi_{k}-\sum_{j=1}^{4} e_{(k, k+2-j)} \hat{\phi}_{k+2-j}\right] /\left[e_{(k, k+2)}\right], \quad \hat{e}_{(k+2, m)}=-\left[\sum_{j=1}^{4} \times\right.$ $\left.e_{(k, k+2-j)} \hat{e}_{(k+2-j, m)}\right] /\left[e_{(k, k+2)}\right]$, for $m=1,2,3,4$, with the conventions $\hat{\phi}_{j}=0, \hat{e}_{(j, j)}=1$, for $j=1,2,3,4, \hat{e}_{(i, j)}=0$, for $i, j=1,2,3,4, i \neq j$, and $e_{(m, i)}=\hat{e}_{(m, j)}=0$, for $\{i, m\}<1$ and $\{i, m\}>n$ and $j=1,2,3,4$, where, for $i=1, \ldots, n, e_{(i, i)}=\left(\rho_{i, 1} b_{i, 1}+c_{i, 1}\right)+\beta(1-2 \alpha)^{2}\left(\rho_{i,}{ }_{2} b_{i, 2}-c_{i, 2}\right)+$ $\beta \alpha^{2}\left[\left(\rho_{i-1,2} b_{i-1,2}-c_{i-1,2}\right)+\left(\rho_{i+1,2} b_{i+1,} \quad 2-c_{i+1,2}\right)\right], \quad e_{(i, i-2)}=\beta \alpha^{2}$ $\left(\rho_{i-1,2} b_{i-1,2}-c_{i-1,2}\right), \quad e_{(i, i-1)}=\beta \alpha(1-2 \alpha)\left[\left(\rho_{i-1,2} b_{i-1,2}-c_{i-1,}, \quad 2\right)+\right.$ $\left.\left(\rho_{i, 2} b_{i, 2}-c_{i, 2}\right)\right], \quad e_{(i, i+1)}=\beta \alpha(1-2 \alpha)\left[\left(\rho_{i, 2} b_{i, 2}-c_{i,} 2\right)+\left(\rho_{i+1,2} b_{i+1,2}-\right.\right.$ $\left.\left.c_{i+1,2}\right)\right], \quad e_{(i, i+2)}=\beta \alpha^{2}\left(\rho_{i+1,2} b_{i+1,2}-c_{i+1,2}\right), e_{(i, j)}=0$, elsewhere and $\phi_{i}=\rho_{i, 1} a_{i, 1}-\beta\left[\alpha\left(\rho_{i-1,2} a_{i-1,2}+c_{i-1,2} w_{1}\right)+(1-2 \alpha)\left(\rho_{i, 2} a_{i, 2}+c_{i, 2} w_{1}\right)+\right.$ $\left.\alpha\left(\rho_{i+1,2} a_{i+1,2}+c_{i+1,2} w_{1}\right)\right]+\beta\left[\alpha \rho_{i-1,2} b_{i-1,2}\left(x_{1}+w_{1}\right)+\right.$ $\left.\rho_{i, 2} b_{i, 2}\left(x_{1}+w_{1}\right)+\alpha \rho_{i+1,2} b_{i+1,2}\left(x_{1}+w_{1}\right)\right]$.

(ii) If $0 \leqslant u_{i, 1}^{* *} \leqslant x_{1}$, for all $i$, then $u_{i, 1}^{* *}$, given above, is the optimal solution for the centralized problem.

For identical users, we find that the results for the optimal solution of the centralized ring configuration are exactly the same as those for the strip configuration, as stated below.

Corollary 8 (Uniqueness of the global maximizer and optimality for identical users). Identical to the results of Corollary 4.

Corollaries 4 and 8 indicate that the configuration of the users does not change the optimal allocation of water among users when the system is managed centrally. In the strip configuration, we have shown that it is not possible to coordinate the system through a centrally set unit cost $\left(c_{t}\right)$. This arises from decentralized decisions of users being non-identical even for identical users due to their differing locations over the common aquifer. For the ring configuration, the decentralized optimal solution is the same for all identical users. The next question we will address is: Is it possible to coordinate the system in the ring configuration?

Corollary 9 (No coordination). In a ring configuration with $n$ identical users, for $\left(\rho_{t} b_{t}+c_{t}\right) x_{0}<\rho_{t} a_{t}<\left(2 \rho_{t} b_{t}+c_{t}\right) x_{0}$, there does not exist a periodic unit pumping $\operatorname{cost} c_{2}$ that equates the Nash equilibrium with the centralized optimal solution, for all $t$.

Thus, under the cost structure adopted herein and by Saak and Peterson [14], the social planner can not entice multiple $(n>2)$ users to behave in accordance with the centralized optimal decision. If the total profits realized from the central allocation of usage are greater than those realized decentrally, then the centralized solution will dominate the decentralized one. Unfortunately, no analytical comparison could be obtained for the total discounted profits realized from the optimal usage quantities under both management systems. However, the following section provides some numerical illustrations and comparisons between the solutions in both configurations.

So far, we have considered the scenario where all water stock is depleted by the end of the problem horizon. Next, we extend this model by allowing users to partially consume water stock for the second period for irrigation purposes and to salvage the remaining stocks according to a quadratic salvage value function. The addition of a salvage function may be viewed as a proxy for the impact of extending the problem horizon. We discuss this variant of the model in the Appendix; we observe that the fundamental results hold under certain conditions for this case, as well.

\section{Illustrative examples}

We next present some numerical examples to illustrate the impact of the number of users and the lateral transmissivity 
Table 1

Equilibrium usage in period 1 for $n$-identical users on a strip.

\begin{tabular}{|c|c|c|c|c|c|c|c|c|c|c|}
\hline$n$ & 1 & 2 & 3 & 4 & 5 & 6 & 7 & 8 & 9 & 10 \\
\hline Setting1 & \multicolumn{10}{|c|}{$\rho_{i, t}=1, a_{i, t}=10, b_{i, t}=5, c_{i, t}=2$} \\
\hline$u_{1,1}^{*}$ & .5 & .6757 & .6631 & .6635 & .6635 & .6635 & .6635 & .6635 & .6635 & .6635 \\
\hline$u_{2,1}^{*}$ & - & .6757 & .8961 & .8890 & .8892 & .8892 & .8892 & .8892 & .8892 & .8892 \\
\hline$u_{3,1}^{*}$ & - & - & .6631 & .8890 & .8819 & .8821 & .8821 & .8821 & .8821 & .8821 \\
\hline$u_{4,1}^{*}$ & - & - & - & .6635 & .8892 & .8821 & .8824 & .8824 & .8824 & .8824 \\
\hline$u_{5,1}^{*}$ & - & - & - & - & .6635 & .8892 & .8821 & .8824 & .8824 & .8824 \\
\hline$u_{6,1}^{*}$ & - & - & - & - & - & .6635 & .8892 & .8821 & .8824 & .8824 \\
\hline$u_{7,1}^{*}$ & - & - & - & - & - & - & .6635 & .8892 & .8821 & .8824 \\
\hline$u_{8,1}^{*}$ & - & - & - & - & - & - & - & .6635 & .8892 & .8821 \\
\hline$u_{9,1}^{*}$ & - & - & - & - & - & - & - & - & .6635 & .8892 \\
\hline$u_{10,1}^{*}$ & - & - & - & - & - & - & - & - & - & .6635 \\
\hline$\widetilde{\Gamma}_{1}^{*}\left(\vec{u}_{1}, \vec{x}_{1}\right)$ & 7.83 & 15.66 & 23.49 & 31.32 & 39.15 & 46.98 & 54.81 & 62.64 & 70.47 & 78.30 \\
\hline$\sum_{i=1}^{n} \Gamma_{i, 1}^{*}\left(\vec{u}_{1}, \vec{x}_{1}\right)$ & 7.83 & 15.20 & 22.26 & 29.28 & 36.08 & 43.12 & 50.14 & 57.16 & 64.18 & 71.20 \\
\hline Setting2 & \multicolumn{10}{|c|}{$\rho_{i, 1}=1.05, \rho_{i, 2}=1, a_{i, t}=10, b_{i, t}=5, c_{i, t}=2$} \\
\hline$u_{1,1}^{*}$ & .5366 & .7105 & 6985 & 6988 & .6988 & .6988 & .6988 & .6988 & 6988 & 6988 \\
\hline$u_{2,1}^{*}$ & - & .7105 & .9274 & .9206 & .9208 & .9208 & .9208 & .9208 & .9208 & .9208 \\
\hline$u_{3,1}^{*}$ & - & - & 6985 & .9206 & .9124 & .9141 & .9141 & .9141 & .9141 & .9141 \\
\hline$u_{4,1}^{*}$ & - & - & - & .6988 & .9208 & .9141 & .9143 & .9143 & .9143 & .9143 \\
\hline$u_{5,1}^{*}$ & - & - & - & - & .6988 & .9208 & .9141 & .9143 & .9143 & .9143 \\
\hline$u_{6,1}^{*}$ & - & - & - & - & - & .6988 & .9208 & .9141 & .9143 & .9143 \\
\hline$u_{7,1}^{*}$ & - & - & - & - & - & - & 6988 & .9208 & .9141 & .9143 \\
\hline$u_{8,1}^{*}$ & - & - & - & - & - & - & - & .6988 & .9208 & .9141 \\
\hline$u_{9,1}^{*}$ & - & - & - & - & - & - & - & - & .6988 & .9208 \\
\hline$u_{10,1}^{*}$ & - & - & - & - & - & - & - & - & - & .6988 \\
\hline$\widetilde{\Gamma}_{1}^{*}\left(\vec{u}_{1}, \vec{x}_{1}\right)$ & 7.98 & 15.96 & 23.94 & 31.92 & 39.90 & 47.88 & 55.86 & 63.84 & 71.82 & 79.80 \\
\hline$\sum_{i=1}^{n} \Gamma_{i, 1}^{*}\left(\vec{u}_{1}, \vec{x}_{1}\right)$ & 7.98 & 15.64 & 21.91 & 28.12 & 35.07 & 42.06 & 49.02 & 55.98 & 62.94 & 69.90 \\
\hline Setting3 & \multicolumn{10}{|c|}{$\rho_{i, t}=1, a_{i, 1}=10.5, a_{i, 2}=10, b_{i, t}=5, c_{i, t}=2$} \\
\hline$u_{1,1}^{*}$ & .55 & .7297 & .7168 & .7172 & .7172 & .7172 & .7172 & .7172 & .7172 & .7172 \\
\hline$u_{2,1}^{*}$ & - & .7297 & .9552 & .9480 & .9482 & .9482 & .9482 & .9482 & .9482 & .9482 \\
\hline$u_{3,1}^{*}$ & - & - & .7168 & .9480 & .9407 & .9410 & .9410 & .9410 & .9410 & .9410 \\
\hline$u_{4,1}^{*}$ & - & - & - & .7172 & .9482 & .9410 & .9412 & .9412 & .9412 & .9412 \\
\hline$u_{5,1}^{*}$ & - & - & - & - & .7172 & .9482 & .9410 & .9412 & .9412 & .9412 \\
\hline$u_{6,1}^{*}$ & - & - & - & - & - & .7172 & .9482 & .9410 & .9412 & .9412 \\
\hline$u_{7,1}^{*}$ & - & - & - & - & - & - & .7172 & .9482 & .9410 & .9412 \\
\hline$u_{8,1}^{*}$ & - & - & - & - & - & - & - & .7172 & .9482 & .9410 \\
\hline$u_{9,1}^{*}$ & - & - & - & - & - & - & - & - & .7172 & .9482 \\
\hline$u_{10,1}^{*}$ & - & - & - & - & - & - & - & - & - & .7172 \\
\hline$\widetilde{\Gamma}_{1}^{*}\left(\vec{u}_{1}, \vec{x}_{1}\right)$ & 8.01 & 16.02 & 24.03 & 32.04 & 40.05 & 48.06 & 56.07 & 64.08 & 72.09 & 80.10 \\
\hline$\sum_{i=1}^{n} \Gamma_{i, 1}^{*}\left(\vec{u}_{1}, \vec{x}_{1}\right)$ & 8.01 & 15.7 & 23 & 30 & 37.49 & 44.73 & 51.96 & 59.21 & 66.46 & 73.71 \\
\hline
\end{tabular}

Table 2

Total equilibrium usage and total profits for $n$-identical users on a strip: time-invariant setting.

\begin{tabular}{|c|c|c|c|c|c|c|c|c|c|c|}
\hline$n$ & 1 & 2 & 3 & 4 & 5 & 6 & 7 & 8 & 9 & 10 \\
\hline$u_{1,1}^{*}+u_{1,2}^{*}$ & 1 & 1 & .9418 & .9436 & .9436 & .9436 & .9436 & .9436 & .9436 & .9436 \\
\hline$u_{2,1}^{*}+u_{2,2}^{*}$ & - & 1 & 1.1165 & 1.0564 & 1.0564 & 1.0582 & 1.0582 & 1.0582 & 1.0582 & 1.0582 \\
\hline$u_{3,1}^{*}+u_{3,2}^{*}$ & - & - & .9418 & 1.0564 & .9928 & .9982 & .9981 & .9981 & .9981 & .9981 \\
\hline$u_{4,1}^{*}+u_{4,2}^{*}$ & - & - & - & .9436 & 1.0564 & .9982 & 1.0002 & 1.0002 & 1.0002 & 1.0002 \\
\hline$u_{5,1}^{*}+u_{5,2}^{*}$ & - & - & - & - & .9436 & 1.0582 & .9982 & 1.0002 & 1.0002 & 1.0002 \\
\hline$u_{6,1}^{*}+u_{6,2}^{*}$ & - & - & - & - & - & .9436 & 1.0582 & .9981 & 1.0002 & 1.0002 \\
\hline$u_{7,1}^{*}+u_{7,2}^{*}$ & - & - & - & - & - & - & .9436 & 1.0582 & .9981 & 1.0002 \\
\hline$u_{8,1}^{*}+u_{8,2}^{*}$ & - & - & - & - & - & - & - & .9436 & 1.0582 & .9981 \\
\hline$u_{9,1}^{*}+u_{9,2}^{*}$ & - & - & - & - & - & - & - & - & .9436 & 1.0582 \\
\hline$u_{10,1}^{*}+u_{10,2}^{*}$ & - & - & - & - & - & - & - & - & - & .9436 \\
\hline$T P_{1}$ & .5 & 1.351 & 2.223 & 3.105 & 3.994 & 4.870 & 5.752 & 6.634 & 7.516 & 8.400 \\
\hline$R_{1} \%$ & 50 & 67.57 & 74.08 & 77.62 & 79.75 & 81.16 & 82.17 & 82.93 & 83.52 & 83.99 \\
\hline
\end{tabular}

coefficient $\alpha$ on the optimal usage quantities and the discounted profits. In all of the following examples, all users are taken as identical with parameters $\beta=1, w_{i, 0}=w_{i, 1}=0$ and $x_{i, 0}=x_{i, 1}=1$. (The numerical results are obtained from the analytical results provided above.) For comparison with the results of Saak and Peterson [14], we assume that $\alpha$ is perceived by all users to be a random variable uniformly distributed over $[0,0.5]$. We provide numerical examples for time-invariant and time-variant settings. 
Table 3

Profits per user in the decentralized problem for $n$-identical users on a strip: time-invariant setting.

\begin{tabular}{|c|c|c|c|c|c|c|c|c|c|c|}
\hline$n$ & 1 & 2 & 3 & 4 & 5 & 6 & 7 & 8 & 9 & 10 \\
\hline$\Gamma_{11}^{*}\left(\vec{u}_{1}, \vec{x}_{1}\right)$ & 7.83 & 7.60 & 7.21 & 7.21 & 7.12 & 7.12 & 7.12 & 7.12 & 7.12 & 7.12 \\
\hline$\Gamma_{2,1}^{*}\left(\vec{u}_{1}, \vec{x}_{1}\right)$ & - & 7.60 & 7.83 & 7.42 & 7.42 & 7.44 & 7.44 & 7.44 & 7.44 & 7.44 \\
\hline$\Gamma_{3,1}^{*}\left(\vec{u}_{1}, \vec{x}_{1}\right)$ & - & - & 7.21 & 7.42 & 7.00 & 7.00 & 7.00 & 7.00 & 7.00 & 7.00 \\
\hline$\Gamma_{4,1}^{*}\left(\vec{u}_{1}, \vec{x}_{1}\right)$ & - & - & - & 7.22 & 7.42 & 7.00 & 7.02 & 7.02 & 7.02 & 7.02 \\
\hline$\Gamma_{5,1}^{*}\left(\vec{u}_{1}, \vec{x}_{1}\right)$ & - & - & - & - & 7.12 & 7.44 & 7.00 & 7.02 & 7.02 & 7.02 \\
\hline$\Gamma_{6,1}^{*}\left(\vec{u}_{1}, \vec{x}_{1}\right)$ & - & - & - & - & - & 7.12 & 7.44 & 7.00 & 7.02 & 7.02 \\
\hline$\Gamma_{7,1}^{*}\left(\vec{u}_{1}, \vec{x}_{1}\right)$ & - & - & - & - & - & - & 7.12 & 7.44 & 7.00 & 7.02 \\
\hline$\Gamma_{8,1}^{*}\left(\vec{u}_{1}, \vec{x}_{1}\right)$ & - & - & - & - & - & - & - & 7.12 & 7.44 & 7.00 \\
\hline$\Gamma_{9,1}^{*}\left(\vec{u}_{1}, \vec{x}_{1}\right)$ & - & - & - & - & - & - & - & - & 7.12 & 7.44 \\
\hline$\Gamma_{10,1}^{*}\left(\vec{u}_{1}, \vec{x}_{1}\right)$ & - & - & - & - & - & - & - & - & - & 7.12 \\
\hline$\sum_{i=1}^{n} \Gamma_{i, 1}^{*}\left(\vec{u}_{1}, \vec{x}_{1}\right)$ & 7.83 & 15.20 & 22.26 & 29.28 & 36.08 & 43.12 & 50.14 & 57.16 & 64.18 & 71.20 \\
\hline
\end{tabular}

Table 4

Equilibrium usage in periods 1 and 2 and total profits for $n$-identical users on a ring.

\begin{tabular}{|c|c|c|c|c|c|c|c|}
\hline$n$ & $u_{i, 1}^{*}$ & $u_{i, 2}^{*}$ & $\left(u_{i, 1}^{*}+u_{i, 2}^{*}\right)$ & $T P_{1}$ & $R_{1} \%$ & $\widetilde{\Gamma}_{1}^{*}\left(\vec{u}_{1}, \vec{x}_{1}\right)$ & $\sum_{i=1}^{n} \Gamma_{i, 1}^{*}\left(\vec{u}_{1}, \vec{x}_{1}\right)$ \\
\hline Setting1 & \multicolumn{7}{|c|}{$\rho_{i, t}=1, a_{i, t}=10, b_{i, t}=5, c_{i, t}=2$} \\
\hline 1 & .5 & .5 & 1 & .5 & 50 & 7.83 & 7.83 \\
\hline 2 & .6757 & .3243 & 1 & 1.3514 & 67.57 & 15.66 & 15.20 \\
\hline$n \geqslant 3$ & .8824 & .1176 & 1 & $.8824 n$ & 88.24 & $7.83 n$ & $7.032 n$ \\
\hline Setting2 & \multicolumn{7}{|c|}{$\rho_{i, 1}=1.05, \rho_{i, 2}=1, a_{i, t}=10, b_{i, t}=5, c_{i, t}=2$} \\
\hline 1 & .5366 & .4634 & 1 & .5366 & 53.66 & 7.98 & 7.98 \\
\hline 2 & .7105 & .2895 & 1 & 1.421 & 71.05 & 15.96 & 15.64 \\
\hline$n \geqslant 3$ & .9143 & .0857 & 1 & $.9143 n$ & 91.43 & $7.98 n$ & $7.24 n$ \\
\hline Setting3 & \multicolumn{7}{|c|}{$\rho_{i, t}=1, a_{i, 1}=10.5, a_{i, 2}=10, b_{i, t}=5, c_{i, t}=2$} \\
\hline 1 & .55 & .45 & 1 & .55 & 55 & 8.01 & 8.01 \\
\hline 2 & .7297 & .2703 & 1 & 1.4594 & 72.97 & 16.02 & 15.7 \\
\hline$n \geqslant 3$ & .9411 & .0589 & 1 & $.9411 n$ & 94.11 & $8.01 n$ & $7.25 n$ \\
\hline
\end{tabular}

Example 1. We first investigate the impact of the number of users on optimal water usage and expected profits in the strip configuration. We consider three different settings in this example. Namely, the first one is time-invariant in which, we set $\rho_{i, t}=1, a_{i, t}=10, b_{i, t}=5$ and $c_{i, t}=2$ for all $i$ and $t$. The second setting is time-variant in which, we set $\rho_{i, 1}=1.05, \rho_{i, 2}=1, a_{i, t}=10, b_{i, t}=5$ and $c_{i, t}=2$ for all $i$ and $t$. The last setting is also time variant in which, we set $\rho_{i, t}=1, a_{i, 1}=10.5$, $a_{i, 2}=10, b_{i, t}=5$ and $c_{i, t}=2$ for all $i$ and $t$. Table 1 summarizes the water usage per user in period 1 accompanied with the total discounted profits in the centralized $\left(\widetilde{\Gamma}_{1}^{*}\right)$ and decentralized $\left(\Gamma_{i, 1}^{*}\right)$ problems realized over the two-period horizon. The centralized solution is found from Corollary 4. More specifically, for timeinvariant setting, we find that $u_{i, 1}^{*}=0.5, i=1, \ldots, n$, and the corresponding discounted profit is 7.83. For $n$ users, the total discounted profit attained by the social planner is $7.83 n$. In the second setting, we have $u_{i, 1}^{*}=0.5366, i=1, \ldots, n$, the discounted profit per user is 7.98 and the total discounted profit of the social planner is $7.98 n$. Likewise, in the last setting, we have $u_{i, 1}^{*}=0.55, i=1, \ldots, n$, the discounted profit per user is 8.01 and total discounted profit attained by the social planner is $8.01 n$. We observe that with higher crop's unit price in period 1 (setting 2 ), users pump more in period 1 and realize more total profits in the centralized problem compared to time-invariant price (setting 1). However, as they pump more under this setting, their total profits in the decentralized problem deteriorate with respect to the time-invariant setting. In setting 3 , we observe that users pump more in period 1 and realize more total profit compared to the time-invariant setting in both centralized and decentralized problems.

Table 2 presents the total usage per user over the two-period planning horizon $\left(u_{i, 1}^{*}+u_{i, 2}^{*}\right)$ of the decentralized problem with time-invariant setting. In this table, $T P_{t}$ denotes the total usage in period $t$ where $\left(T P_{t}=\sum_{i=1}^{n} u_{i, t}^{*}\right)$ and $R_{t} \%$ denotes the percentage of the average usage, $\left(R_{t} \%=\left(T P_{t} / n\right) \times 100 \%\right)$. The optimal water usage is symmetric around the mid-point of the strip but not monotone with respect to the user location. This numerically validates Saak and Peterson's [14] conjecture as noted in Section 3.1. We make two observations. (i) Non-extreme users pump more than the extreme ones in period 1, while the opposite is true in period 2. (ii) The total water usage may exceed the initial stock levels for some users. Table 3 tabulates the total discounted profit per user in the decentralized problem. We note that the profits are consistent with the total water usage; that is, highest profits are obtained by the second to extreme users. Likewise, profits are also symmetric around the midpoints and non-monotone. However, the least profits are not realized by the extreme users, which may be attributed to the non-linear nature of the profit function. It is worth noting that, under the time-variant settings, users exhibit the same behavior in pumpage and in profit realization, and, hence, we skip giving their results.

Example 2. We now consider the ring configuration with the same settings above. Table 4 summarizes the corresponding numerical results. We observe that users pump more and realize more profits under the time-variant settings compared to the time-invariant one. The corresponding centralized solutions are found from Corollary 8 , which are the same as those found in Corollary 4 above in the strip configuration. Fig. 1a depicts the values of $R_{1} \%$ versus the number of users $n$ for strip and ring configurations for the data tabulated in Tables 1 and 4 corresponding to the timeinvariant setting. We observe that $R_{1} \%$ increases concavely in the number of users. This implies that users become more greedy as more users share the resource, however the tendency to pump more water diminishes. As expected, for both configurations, the 


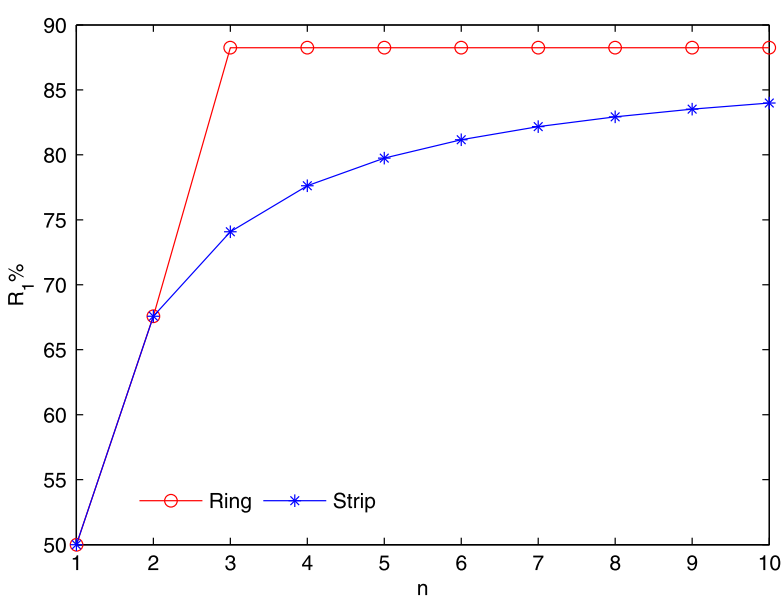

(a) $R_{1} \%$ vs. $n$

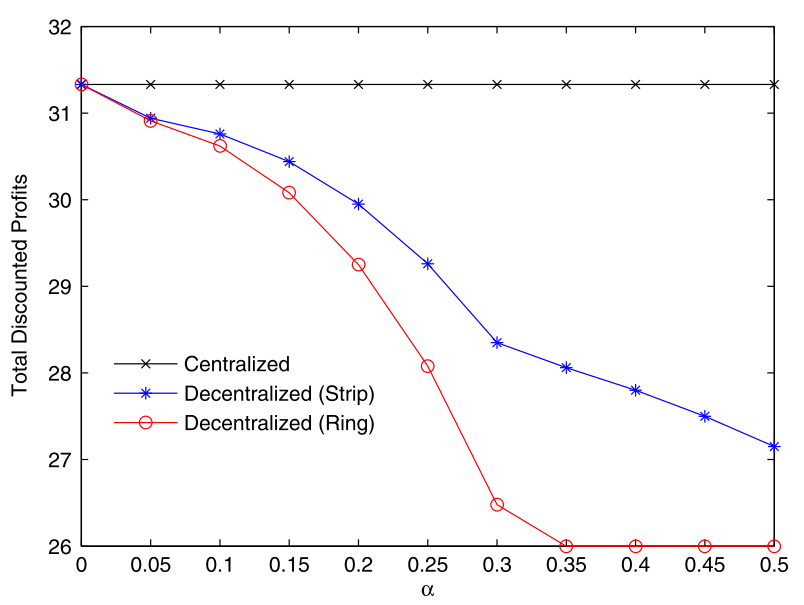

(b) Identical case $(n=4)$ : Total discounted profit vs. $\alpha$

Fig. 1. $\left(R_{1} \%\right.$ vs. $\left.n\right)$, (Total discounted profits vs. $\alpha$ ): time-invariant setting.

maximum discounted profits are attained in the centralized problem. However, for $n \geqslant 3$, the strip configuration yields more discounted profits than the ring configuration in the decentralized problem. This occurs because, users in the strip configuration exhibit an oscillating greedy behavior of pumping in period 1 where they pump more water than they do in the ring configuration. Again, it is worth noting that users show the same behavior in their $R_{1} \%$ under the time-variant settings and, hence, their corresponding figures are not given.
Example 3. In this example, we examine the effect of $\alpha \in[0,0.5]$ on the total decentralized discounted profits in both configurations for $n=4$ identical users. Here, we assume that users have perfect information about the soil transmissivity and treat $\alpha$ as a deterministic parameter. We set $\rho_{i, t}=1, a_{i, t}=10, b_{i, t}=5$ and $c_{i, t}=2$ for all $i$ and $t$, (i.e., the time-invariant setting). Tables 5 and 6 summarize the results for the strip and ring configurations, respectively. In both tables, $\Delta P \%=\left[\left(\widetilde{\Gamma}_{1}^{*}-\Gamma_{i, 1}^{*}\right)\right] / \widetilde{\Gamma}_{1}^{*} \times 100 \%$ stands for the percentage rate of decrease in discounted profit of the decentralized problem relative to that in the centralized problem. In the strip configuration, the unconstrained solution for $\alpha \in[0.35,0.5]$, resulted in infeasible solutions; $u_{2,1}^{* *}=u_{3,1}^{* *}>1$ and $u_{1,1}^{* *}=u_{4,1}^{* *}<1$. Hence, we obtained the constrained solution numerically, $u_{2,1}^{*}=u_{3,1}^{*}=1$, (i.e. $\delta_{2}^{*}=\delta_{3}^{*}>0$ ) and $u_{1,1}^{*}=u_{4,1}^{*}<1$, (i.e. $\delta_{1}^{*}=\delta_{4}^{*}=0$ ). Similarly, the unconstrained solutions are suboptimal for the ring configuration for $\alpha \in[0.35,0.5]$. The optimal solution obtained numerically results in all users are depleting their total available stock of water in period $1, u_{i, 1}^{*}=1$ and $\delta_{i}^{*}=0$, for $i=1,2,3,4$. We note that in both configurations, as $\alpha$ increases, users experience more effects of hydrologic dynamics and become more greedy tending to use more water in period 1 . Fig. $1 \mathrm{~b}$ depicts the total discounted profits with respect to $\alpha$ in both configurations. As observed from the figure, the total discounted profits are non-increasing in $\alpha$ regardless of the configuration. However, the rate of decrease, $\Delta P \%$, in the strip configuration is always lower than that in the ring for $\alpha \in[0,0.50]$. It is important to note that in both configurations, the maximum discounted profit is attained in the centralized setting where the realized total discounted profit is 31.33 . However, both centralized and decentralized problems achieve the same value of total discounted profits when there is no lateral flow between users (i.e., when $\alpha=0)$, as expected.

\section{Conclusions}

In this work, we consider ground water usage when the resources are shared among $n$ users under centralized and decentralized management settings. Our work extends the results of Saak and Peterson [14] to $n$ non-identical users by considering two different user configurations - strip and ring - overlying a common groundwater aquifer. It is assumed that transmission of the groundwater is governed by Darcy's Law, which induces a special interaction type among the users between the periods. For a quadratic periodic profit function, general analytical solutions related to the optimal Nash equilibrium usage for the decentralized problem are obtained for both strip and ring transmission configurations for a two-period planning horizon. Also, we are able to arrive at more compact analytical results for the special case of identical users for the centralized and the decentralized problems in both configurations. However, in both configurations, the

Table 5

Total discounted profit vs. $\alpha$ : strip configuration.

\begin{tabular}{|c|c|c|c|c|c|c|c|}
\hline$\alpha$ & $\left(u_{1,1}^{*}, u_{1,2}^{*}\right)$ & $\left(u_{2,1}^{*}, u_{2,2}^{*}\right)$ & $\left(u_{3,1}^{*}, u_{3,2}^{*}\right)$ & $\left(u_{4,1}^{*}, u_{4,2}^{*}\right)$ & $\widetilde{\Gamma}_{1}^{*}\left(\vec{u}_{1}, \vec{x}_{1}\right)$ & $\sum_{i=1}^{n} \Gamma_{i, 1}^{*}\left(\vec{u}_{1}, \vec{x}_{1}\right)$ & $\triangle P \%$ \\
\hline 0 & $(.5, .5)$ & $(.5, .5)$ & $(.5, .5)$ & $(.5, .5)$ & 31.33 & 31.33 & 0 \\
\hline .05 & $(.5325, .4658)$ & $(.5675, .4343)$ & $(.5675, .4343)$ & $(.5325, .4658)$ & 31.33 & 30.94 & 1.25 \\
\hline .10 & $(.5649, .4276)$ & $(.6402, .3673)$ & $(.6402, .3673)$ & $(.5649, .4276)$ & 31.33 & 30.76 & 1.82 \\
\hline .15 & $(.5972, .3846)$ & $(.7185, .2997)$ & $(.7185, .2997)$ & $(.5972, .3846)$ & 31.33 & 30.44 & 2.84 \\
\hline .20 & $(.6295, .3359)$ & $(.8025, .2321)$ & $(.8025, .2321)$ & $(.6295, .3359)$ & 31.33 & 29.95 & 4.4 \\
\hline .25 & $(.6616, .2807)$ & $(.8925, .1652)$ & $(.8925, .1652)$ & $(.6616, .2807)$ & 31.33 & 29.26 & 6.6 \\
\hline .30 & $(.6939, .2177)$ & $(.9885, .0999)$ & $(.9885, .0999)$ & $(.6939, .2177)$ & 31.33 & 28.35 & 9.51 \\
\hline .35 & $(.7339, .1729)$ & $(1, .0931)$ & $(1, .0931)$ & $(.7339, .1729)$ & 31.33 & 28.06 & 10.4 \\
\hline .40 & $(.7772, .1337)$ & $(1, .0891)$ & $(1, .0891)$ & $(.7772, .1337)$ & 31.33 & 27.80 & 11.27 \\
\hline .45 & $(.8230, .0974)$ & $(1, .0797)$ & $(1, .0797)$ & $(.8230, .0974)$ & 31.33 & 27.50 & 12.22 \\
\hline .50 & $(.8709, .0646)$ & $(1, .0646)$ & $(1, .0646)$ & $(.8709, .0646)$ & 31.33 & 27.15 & 13.34 \\
\hline
\end{tabular}


Table 6

Total discounted profit vs. $\alpha$ : ring configuration.

\begin{tabular}{|c|c|c|c|c|c|c|c|}
\hline$\alpha$ & $\left(u_{1,1}^{*}, u_{1,2}^{*}\right)$ & $\left(u_{2,1}^{*}, u_{2,2}^{*}\right)$ & $\left(u_{3,1}^{*}, u_{3,2}^{*}\right)$ & $\left(u_{4,1}^{*}, u_{4,2}^{*}\right)$ & $\widetilde{\Gamma}_{1}^{*}\left(\vec{u}_{1}, \vec{x}_{1}\right)$ & $\sum_{i=1}^{n} \Gamma_{i, 1}^{*}\left(\vec{u}_{1}, \vec{x}_{1}\right)$ & $\triangle P \%$ \\
\hline 0 & $(.5, .5)$ & $(.5, .5)$ & $(.5, .5)$ & $(.5, .5)$ & 31.33 & 31.33 & 0 \\
\hline .05 & $(.5670, .4330)$ & $(.5670, .4330)$ & $(.5670, .4330)$ & $(.5670, .4330)$ & 31.33 & 30.91 & 1.34 \\
\hline .10 & $(.6383, .3617)$ & $(.6383, .3617)$ & $(.6383, .3617)$ & $(.6383, .3617)$ & 31.33 & 30.62 & 2.17 \\
\hline .15 & $(.7143, .2857)$ & $(.7143, .2857)$ & $(.7143, .2857)$ & $(.7143, .2857)$ & 31.33 & 30.08 & 4 \\
\hline .20 & $(.7955, .2045)$ & $(.7955, .2045)$ & $(.7955, .2045)$ & $(.7955, .2045)$ & 31.33 & 29.25 & 6.64 \\
\hline .25 & $(.8824, .1176)$ & $(.8824, .1176)$ & $(.8824, .1176)$ & $(.8824, .1176)$ & 31.33 & 28.08 & 10.4 \\
\hline .30 & $(.9756, .0244)$ & $(.9756, .0244)$ & $(.9756, .0244)$ & $(.9756, .0244)$ & 31.33 & 26.48 & 15.5 \\
\hline .35 & $(1,0)$ & $(1,0)$ & $(1,0)$ & $(1,0)$ & 31.33 & 26 & 17 \\
\hline .40 & $(1,0)$ & $(1,0)$ & $(1,0)$ & $(1,0)$ & 31.33 & 26 & 17 \\
\hline .45 & $(1,0)$ & $(1,0)$ & $(1,0)$ & $(1,0)$ & 31.33 & 26 & 17 \\
\hline .50 & $(1,0)$ & $(1,0)$ & $(1,0)$ & $(1,0)$ & 31.33 & 26 & 17 \\
\hline
\end{tabular}

centralized solution can not be achieved in the decentralized game-theoretic setting through a single pricing mechanism (i.e. no coordination). Our analytical results reveal that in strip configuration with identical users, the optimal Nash equilibrium usage quantities oscillate about the optimal Nash equilibrium usage quantities of the ring configuration. We also note that although the optimal solutions of the strip structure do not converge to that of the ring structure as the number of users increase, they are observed to become very close in our numerical examples for the non-extreme users of the strip. In our numerical results of timeinvariant setting, we observe that, in both strip and ring configurations in decentralized problems, as the underground water transmission coefficient increases, users become more greedy and use more water. This greedy behavior however adversely affects the system's total discounted profit. On the other hand, we investigate the water pumping behavior of users under the time-variant setting by varying one parameter at a time and keeping the rest as in the time-invariant setting. In particular, we study the effect of changing the crop unit price and yield function parameters on the optimal solution as well as on the realized total profits in the centralized and decentralized problems. In all settings (variant and invariant), the centralized solutions always dominate the decentralized ones by achieving more profits.

In the presence of a salvage function for leftover water stock at the end of problem horizon, we observe that, in both configurations, the centralized solution dominates the decentralized one by realizing more profits from water usage. Also, in the strip configuration, water usage fluctuates from ends toward the midpoints of the strip. Additionally, in both configurations and in both problems, users allocate part of their available water stocks in the second period to satisfy demands other than the irrigation ones through selling it out according to the given quadratic salvage value function. In the sequel, under this setting, the policy makers (users and social planner) have more flexibility in allocating their water stock in the second period among two different sources of water demand. Our findings fit within the broader literature on management and operating policy making for usage of limited natural resources. We hope that, in the context of groundwater management, our results will aid the decision makers in developing and adopting control policies for more effective and fair usage of limited resources.

\section{Acknowledgment}

The authors thank The Scientific and Technological Research Council of Turkey for supporting this research work.

\section{Appendix A. A model with salvage}

In this section, we incorporate into our models the possibility of salvaging remaining stock. We assume that it is not necessary for the available stock of water at the beginning of period $2, x_{i, 2}$, to be completely consumed in irrigation. More specifically, part of $x_{i, 2}$ which represents the pumpage quantity in period $2, u_{i, 2}$, is used to satisfy irrigation demands while the remaining part, $\left(x_{i, 2}-u_{i, 2}\right)$, is salvaged. We assume a quadratic salvage value function for the unused water quantity in period $2,\left(x_{i, 2}-u_{i, 2}\right)$, for $i=1, \ldots, n$, given by

$s v_{i, 2}\left(u_{i, 2}, x_{i, 2}\right)=f_{i, 1}\left(x_{i, 2}-u_{i, 2}\right)-0.5 f_{i, 2}\left(x_{i, 2}-u_{i, 2}\right)^{2}$,

where $f_{i, t}$ is positive and allowed to vary over time, $i=1, \ldots, n, t=1$, 2 . Now, we let $\tilde{g}_{i, 2}\left(u_{i, 2}, x_{i, 2}\right)=g_{i, 2}\left(u_{i, 2}, x_{i, 2}\right)+s v_{i, 2}\left(u_{i, 2}, x_{i, 2}\right)$. To find the optimal water pumpage quantity $u_{i, 2}^{*}$, we optimize $\tilde{g}_{i, 2}\left(u_{i, 2}, x_{i, 2}\right)$ with respect to $u_{i, 2}$. Specifically, we find the unconstrained solution of $\tilde{g}_{i, 2}\left(u_{i, 2}, x_{i, 2}\right)$ and determine its feasibility conditions. The unconstrained solution is found by solving the FOC of $\tilde{g}_{i, 2}\left(u_{i, 2}, x_{i, 2}\right)$. From $\partial \tilde{g}_{i, 2}(\cdot, \cdot) / \partial u_{i, 2}=\partial g_{i, 2}(\cdot, \cdot) / \partial u_{i, 2}+\partial s v_{i, 2}(\cdot, \cdot) / \partial u_{i, 2}=0$, the unconstrained solution is given by

$u_{i, 2}^{* *}=\frac{\rho_{i, 2} a_{i, 2}+\left(c_{i, 2}+f_{i, 2}\right) x_{i, 2}-c_{i, 2} x_{i, 0}-f_{i, 1}}{\rho_{i, 2} b_{i, 2}+c_{i, 2}+f_{i, 2}}$.

To guarantee the feasibility of Eq. (17), the following condition should hold

$$
\begin{aligned}
c_{i, 2} x_{i, 0}+f_{i, 1}-\left(c_{i, 2}+f_{i, 2}\right) x_{i, 2} & <\rho_{i, 2} a_{i, 2} \\
& <c_{i, 2} x_{i, 0}+f_{i, 1}+\rho_{i, 2} b_{i, 2} x_{i, 2} .
\end{aligned}
$$

If Eq. (18) holds, then $u_{i, 2}^{*}=u_{i, 2}^{* *}$, for $i=1, \ldots, n$. We observe that $g_{i, 1}\left(u_{i, 1}, x_{i, 1}\right)$ is concave in $u_{i, 1}$ since $\partial^{2} g_{i, 1}(\cdot, \cdot) / \partial\left(u_{i, 1}\right)^{2}=-\left(\rho_{i, 1} b_{i, 1}+-\right.$ $\left.c_{i, 1}\right)<0, \quad i=1, \ldots, n$. Also, we notice that $\partial^{2} \tilde{g}_{i, 2}(\cdot, \cdot) / \partial\left(u_{i, 2}\right)^{2}=-$ $\left(\rho_{i, 2} b_{i, 2}+c_{i, 2}+f_{i, 2}\right)<0$, implying that $\tilde{g}_{i, 2}\left(u_{i, 2}, x_{i, 2}\right)$ is concave in $u_{i, 2}$, $i=1, \ldots, n$. Therefore, $g_{i, 1}\left(u_{i, 1}, x_{i, 1}\right)$ and $\tilde{g}_{i, 2}\left(u_{i, 2}, x_{i, 2}\right)$ are continuous and concave in their respective decision variables, for all $i$. To avoid repetition of similar results in the original model, we only present the changes that might appear under this new model in the results of the strip and ring configurations.

\section{A.1. Strip configuration: the decentralized problem}

For $t=1,2$, and $i=1, \ldots, n$, the decentralized problem of user $i$ is given by

$\left.\Gamma_{i, 1}^{*}\left(\vec{u}_{1}, \vec{x}_{1}\right)=\max _{u_{i, 1}} \Gamma_{i, 1}\left(\vec{u}_{1}, \vec{x}_{1}\right)=\max _{u_{i, 1}}\left[g_{i, 1}\left(u_{i, 1}, x_{i, 1}\right)+\beta \tilde{g}_{i, 2}\left(u_{i, 2}^{* *}, x_{i, 2}(1)\right)\right]\right)$ s.t. (4) and (5)

The function $\Gamma_{i, 1}\left(\vec{u}_{1}, \vec{x}_{1}\right)$ is continuous and jointly concave in $\vec{u}_{1}$ if and only if the following condition holds for all $i$

$$
\begin{aligned}
& 2 c_{i, 2}\left(c_{i, 2}+f_{i, 2}\right)\left(\rho_{i, 2} b_{i, 2}+c_{i, 2}+f_{i, 2}\right)-\left(\rho_{i, 2} b_{i, 2}\right)^{2} f_{i, 2}-\left(c_{i, 2}+f_{i, 2}\right) \\
& \quad \times\left(\rho_{i, 2} b_{i, 2}+c_{i, 2}\right) \leqslant 0 .
\end{aligned}
$$

Proposition 2 of the original model holds for this model. Also, Proposition 3 holds with 
$\gamma_{i}=\left\{\begin{array}{ll}\frac{\beta(1-\alpha)^{2}}{y^{2}} z-\left(\rho_{i, 1} b_{i, 1}+c_{i, 1}\right), & i=1, n, \\ \frac{\beta(1-2 \alpha)^{2}}{y^{2}} z-\left(\rho_{i, 1} b_{i, 1}+c_{i, 1}\right), & \text { o.w. }\end{array}\right.$,

$\sigma_{i}=\left\{\begin{array}{ll}\frac{\beta \alpha(1-\alpha)}{y^{2}} z, & i=1 \\ \frac{\beta \alpha(1-2 \alpha)}{y^{2}} z, & \text { o.w. }\end{array}\right.$,

$\epsilon_{i}=\left\{\begin{array}{ll}\frac{\beta \alpha(1-\alpha)}{y^{2}} z, & i=n-1, \\ \frac{\beta \alpha(1-2 \alpha)}{y^{2}} z, & \text { o.w. }\end{array}\right.$ and

$\lambda_{i}= \begin{cases}\frac{\beta(1-\alpha)}{y^{2}}\left[v_{0} y+v_{1}\right]-\rho_{i, 1} a_{i, 1}, & i=1, n, \\ \frac{\beta(1-2 \alpha)}{y^{2}}\left[v_{0} y+v_{1}\right]-\rho_{i, 1} a_{i, 1}, & \text { o.w. }\end{cases}$

where, for $i=1, \ldots, n, \quad y=\rho_{i, 2} b_{i, 2}+c_{i, 2}+f_{i, 2}, \quad z=\left(c_{i, 2}+f_{i, 2}\right)\left[2 c_{i, 2} y-\right.$ $\left.\left(c_{i, 2}+f_{i, 2}\right)\left(\rho_{i, 2} b_{i, 2}+c_{i, 2}\right)\right]-\left(\rho_{i, 2} b_{i, 2}\right)^{2} f_{i, 2}, v_{0}=\left(2 c_{i, 2}+f_{i, 2}\right) \rho_{i, 2} a_{i, 2}+2 c_{i, 2}-$ $\left(c_{i, 2}+f_{i, 2}\right) w_{1}+c_{i, 2}\left(f_{i, 2} x_{i, 0}-f_{i, 1}\right) \quad$ and $\quad v_{1}=\rho_{i, 2} b_{i, 2}\left[f_{i, 1}\left(\rho_{i, 2} b_{i, 2}+c_{i, 2}\right)-\right.$ $\left.\rho_{i, 2} b_{i, 2} f_{i, 2}\left(x_{i, 0}+w_{1}\right)+\rho_{i, 2} a_{i, 2} f_{i, 2}-c_{i, 2} f_{i, 2} x_{i, 0}\right]-\left(\rho_{i, 2} b_{i, 2}+c_{i, 2}\right)\left(c_{i, 2}+f_{i, 2}\right)$ $\left[\rho_{i, 2} a_{i, 2}+f_{i, 2} x_{i, 0}+\left(c_{i, 2}+f_{i, 2}\right) w_{1}-f_{i, 1}\right]$. Accordingly, Corollary 2 holds with $\gamma=\frac{\beta(1-\alpha)^{2}}{y^{2}} z-\left(\rho_{1} b_{1}+c_{1}\right), \epsilon=\frac{\beta(1-2 \alpha)^{2}}{y^{2}} z-\left(\rho_{1} b_{1}+c_{1}\right), \omega=\frac{\beta \alpha(1-\alpha)}{y^{2}} z$, $\sigma=\frac{\beta \alpha(1-2 \alpha)}{y^{2}} z, \eta=\frac{\beta(1-\alpha)}{y^{2}}\left[v_{0} y+v_{1}\right]-\rho_{1} a_{1}, \lambda=\frac{\beta(1-2 \alpha)}{y^{2}}\left[v_{0} y+v_{1}\right]-\rho_{1} a_{1}, y=$ $\rho_{2} b_{2}+c_{2}+f_{2}, z=\left(c_{2}+f_{2}\right)\left[2 c_{2} y-\left(c_{2}+f_{2}\right)\left(\rho_{2} b_{2}+c_{2}\right)\right]-\left(\rho_{2} b_{2}\right)^{2} f_{2}, v_{0}=$ $\left(2 c_{2}+f_{2}\right) \rho_{2} a_{2}+2 c_{2}\left(c_{2}+f_{2}\right) w_{1}+c_{2}\left(f_{2} x_{0}-f_{1}\right)$ and $v_{1}=\rho_{2} b_{2}\left[f_{1}\left(\rho_{2} b_{2}+\right.\right.$ $\left.\left.c_{2}\right)-\rho_{2} b_{2} f_{2}\left(x_{0}+w_{1}\right)+\rho_{2} a_{2} f_{2}-c_{2} f_{2} x_{0}\right]-\left(\rho_{2} b_{2}+c_{2}\right)\left(c_{2}+f_{2}\right) \quad\left[\rho_{2} a_{2}+\right.$ $\left.f_{2} x_{0}+\left(c_{2}+f_{2}\right) w_{1}-f_{1}\right]$.

\section{A.2. Strip configuration: the centralized problem}

The centralized problem under this setting has the same form of that given in Eq. (12) subject to the constraints in Eqs. (4) and (7). Similarly, if the condition in Eq. (20) holds, then $\widetilde{\Gamma}_{1}\left(\vec{u}_{1}, \vec{x}_{1}\right)$ is continuous and jointly concave in $\vec{u}_{1}$, for all $i$. Fundamentally, Proposition 4 of the original model holds under this setting. However, the coefficients $e_{(i, i)}$ and the right hand sides $\theta_{i}$, for $i=1, \ldots, n$, will be very messy, and, hence, we skip writing their formulae. For identical users, the elements of matrix $\widetilde{A}$ and the right hand side $\widetilde{W}$ in Section 3.2 become $\phi_{1}=-\left(\rho_{1} b_{1}+c_{1}\right)+\beta\left(1-2 \alpha+2 \alpha^{2}\right) \frac{\tilde{z}}{y}, \quad \phi_{2}=\beta(2 \alpha-$ $\left.3 \alpha^{2}\right) \frac{\tilde{z}}{y}, \phi_{3}=\beta \alpha^{2} \frac{\tilde{z}}{y}, \omega_{1}=-\left(\rho_{1} b_{1}+c_{1}\right)+\beta\left(1-4 \alpha+6 \alpha^{2}\right) \frac{\tilde{z}}{y}, \omega_{2}=\phi_{1}-$ $\omega_{1}=2 \beta \alpha(1-2 \alpha) \frac{\tilde{z}}{y}$ and $\theta=\beta \frac{\tilde{y}}{y}-\rho_{1} a_{1}$, where $y=\rho_{2} b_{2}+c_{2}+f_{2}$, $\tilde{z}=y\left[\left(c_{2}+1\right)\left(c_{2}+f_{2}\right) y+\left(\rho_{2} b_{2}+c_{2}\right)\left(c_{2}+f_{2}\right)^{2}\right]-\left(\rho_{2} b_{2}\right)^{2} f_{2}$ and $\tilde{y}=$ $\left(c_{2}+f_{2}\right)\left(\rho_{2} a_{2}-c_{2} w_{1}\right)+\left(\rho_{2} a_{2}+f_{2} x_{1}-c_{2} w_{1}-f_{1}\right)\left[1+\left(c_{2}+f_{2}\right)\left(\rho_{2} b_{2}+\right.\right.$ $\left.\left.c_{2}\right)\right]-\rho_{2} b_{2} f_{1}-\frac{f_{2}}{y}\left[\rho_{2} a_{2}-\rho_{2} b_{2}-c_{2}\left(x_{1}+w_{1}\right)-f_{1}\right]$. The following result gives the solution of the centralized problem for identical users which is equivalent to Corollary 4 and, hence, its proof is omitted.

Corollary 10. Uniqueness of the global maximizer and optimality for identical users).

(i) Suppose that users are identical. Then, $\widetilde{\Gamma}_{1}\left(\vec{u}_{1}, \vec{x}_{1}\right)$ is unique and given by

$u_{i, 1}^{* *}=u^{* *}=\left[\beta \tilde{y}-\rho_{1} a_{1} y\right] /\left[\beta \tilde{z}-\left(\rho_{1} b_{1}+c_{1}\right) y\right], \quad \forall i$.

(ii) If $0 \leqslant u_{i, 1}^{* *} \leqslant x_{1}$, for all $i$, then the optimal solution for the centralized problem is given by $u^{* *}$ above.

Corollary 5 holds as well in this setting.

\section{A.3. Ring configuration: the decentralized problem}

Similar to the strip's decentralized problem, $\Gamma_{i, 1}\left(\vec{u}_{1}, \vec{x}_{1}\right)$ is continuous and jointly concave in $\vec{u}_{1}$ if and only if the condition in Eq. (20) is satisfied for all $i$. Proposition 6 hold under this setting as well. Also, Proposition 7 holds with $\epsilon_{i}=\frac{\beta(1-2 \alpha)^{2}}{y^{2}} z-\left(\rho_{i, 1} b_{i, 1}+c_{i, 1}\right), \sigma_{i}=\frac{\beta \alpha(1-2 \alpha)}{y^{2}} z$, $\lambda_{i}=\frac{\beta(1-2 \alpha)}{y^{2}}\left[v_{0} y+v_{1}\right]-\rho_{i, 1} a_{i, 1}$, and $y, z, v_{0}$ and $v_{1}$ are as defined before in the strip configuration. Furthermore, Corollary 6 holds in this setting.

\section{A.4. Ring configuration: the centralized problem}

Similar to the strip's centralized problem, if the condition in Eq. (20) holds, then $\widetilde{\Gamma}_{1}\left(\vec{u}_{1}, \vec{x}_{1}\right)$ is continuous and jointly concave in $\vec{u}_{1}$, for all $i$. Fundamentally, Proposition 8 of the original model holds under this setting. However, the coefficients $e_{(i, i)}$ and the right hand sides $\phi_{i}$, for $i=1, \ldots, n$, will be very messy, and, hence, we skip writing their formulae. In the identical case, we observe that the elements of matrix $\widetilde{B}$ in Section 4.2 are $\phi_{i}, i=1,2,3, \omega_{i}, i=1,2$ and $\theta$ are as defined above in matrix $\widetilde{A}$ of the strip configuration. The optimal solution of the centralized problem corresponding to the ring configuration give the same solution given in Corollary 10 . Corollary 9 holds under this setting as well.

\section{Appendix B. Supplementary data}

Supplementary data associated with this article can be found, in the online version, at doi:10.1016/j.ejor.2011.05.048.

\section{References}

[1] R. Allen, M. Gisser, Competition versus optimal control in groundwater pumping when demand is nonlinear, Water Resources Research 20 (7) (1984) 752-756.

[2] M. Azaiez, M. Hariga, A single-period model for conjunctive use of ground and surface water under severe overdrafts and water deficit, European Journal of Operational Research 133 (2001) 653-666.

[3] M. Azaiez, A model for conjunctive use of ground and surface water with opportunity costs, European Journal of Operational Research 142 (2002) 611624.

[4] M. Azaiez, M. Hariga, M. Al-Harkan, A chance-constrained multi-period model for a special multi-reservoir system, Computer and Operations Research 32 (2005) 1337-1351.

[5] P. Dasgubta, E. Maskin, The existence of equilibrium in discontinuous economic games, I: theory, Review of Economic Studies 53 (1986) 1-26.

[6] S. Elaydi, An Introduction to Difference Equations, 3rd ed., Springer, United Kingdom, 2005. Chapter 2.

[7] M. Gisser, D. Sanchez, Competitive versus optimal control in groundwater pumping, Water Resources Research 16 (4) (1980) 638-642.

[8] M. Haouari, M. Azaiez, Optimal cropping patterns under water deficits, European Journal of Operational Research 130 (2001) 133-146.

[9] G. Hornberger, P. Wiberg, J. Eshleman, Elements of Physical Hydrology, John Hopkins University Press, Baltimore, 1998 (Chapter 6).

[10] J. Labadie, Optimal operations of multireservoir systems: state-of-the-art review, Water Resources Research 130 (2) (2004) 93-111.

[11] D. Negri, The common property aquifer as a differential game, Water Resources Research 25 (1) (1989) 9-15.

[12] J. Nocedal, S. Wright, Numerical Optimization, 2nd ed., Springer Series in Operations Research, United States of America, 2006 (Chapter 16).

[13] J. Peterson, Y. Ding, Economic adjustments to groundwater depletion in the high plains: do water-saving irrigation systems save water?, American Journal of Agricultural Economics 87 (1) (2005) 147-159

[14] A. Saak, J. Peterson, Groundwater use under incomplete information, Journal of Environmental Economics and Management 54 (2007) 214-228.

[15] W. Yeh, Reservoir management and operations models: state-of-the-art review, Water Resources Research 21 (12) (1985) 1797-1818. 